\title{
A Nonconvex Nonsmooth Regularization Method for Compressed Sensing and Low Rank Matrix Completion ${ }^{\text {tr }}$
}

\author{
Zhuo-Xu Cui, Qibin Fan* \\ School of Mathematics and Statistics, Wuhan University, Wuhan 430072, P.R. China
}

\begin{abstract}
In this paper, a nonconvex and nonsmooth method for compressed sensing and lowrank matrix completion is studied. The proposed model is formulated as nonconvex regularized least square optimization problem. At first, an alternating minimization scheme is developed in which the problem can be decomposed into three subproblems, two of them are convex and the remaining one is smooth. Then, the convergence of the sequence which is generated by the alternating minimization algorithm is proved. In addition, some recovery guarantees are also analysed. Finally, various numerical simulations are performed to test the efficiency of the method.

Keywords: Compressed sensing, low rank matrix completion, nonconvex nonsmooth regularization, alternating minimization method.
\end{abstract}

\section{Introduction}

The compressed sensing (CS) problem is adopted to recover an unknown vector from a small amount of observations. It's possible to reconstruct it exactly with high probability when the vector is sparse [5]. The mathematical formula reads:

$$
\min _{\mathbf{x} \in \mathbb{R}^{p}}\left\{\|\mathbf{x}\|_{0}: A \mathbf{x}=\mathbf{y}\right\}
$$

This work is supported by the National Science Foundation of China under Grant 61179039 and the National Key Basic Research Development Program (973 Program) of China under Grant 2011CB707100.

* Corresponding author

Email addresses: zhuoxucui@whu.edu.cn (Zhuo-Xu Cui), qbfan@whu.edu.cn (Qibin Fan) 
where $\mathbf{y} \in \mathbb{R}^{n}$ with $n \ll p, A \in \mathbb{R}^{n \times p}$ is a measurement ensemble $[7,5,6,8,10]$ and the $\ell_{0}$-norm is defined by

$$
\|\mathbf{x}\|_{0}=\sharp\left\{x_{i}, i=1, \ldots, p: x_{i} \neq 0\right\},
$$

where $\sharp$ denotes cardinality. The matrix completion (MC) problem is to recover a lowrank matrix from a small amount of observations:

$$
\min _{X \in \mathbb{R}^{n_{1} \times n_{2}}}\{\operatorname{rank}(X): \mathcal{A} X=\mathbf{b}\}
$$

where $\mathcal{A}$ is a linear operator and $\mathbf{b} \in \mathbb{R}^{n_{3}}$. In general, we choose $\mathcal{A}$ as a componentwise projection $[4,9]$.

Due to the minimization of $\ell_{0}$-norm regularized problem (1.1) and rank function (1.2) are NP-hard problems in general [31], in some sense, $\ell_{1}$-norm and nuclear norm are the tightest convex relaxation of these nonconvex functions, respectively [22], where

${ }_{12} \ell_{1}$-norm of $\mathbf{x}$ in $\mathbb{R}^{d}$ is defined as $\|\mathbf{x}\|_{1}=\sum_{i=1}^{p}\left|x_{i}\right|$ and the nuclear norm of $X$ in $\mathbb{R}^{n_{1} \times n_{2}}$ is defined as $\|X\|_{*}=\sum_{i=1}^{m} \sigma_{i}(X)$, where $\sigma_{i}$ is the $i$ th largest singular value of $X$ and $m=\min \left\{n_{1}, n_{2}\right\}$. Therefore, the problems (1.1) and (1.2) can be relaxed into:

$$
\begin{gathered}
\min _{\mathbf{x} \in \mathbb{R}^{p}}\left\{\|\mathbf{x}\|_{1}: A \mathbf{x}=\mathbf{y}\right\}, \\
\min _{X \in \mathbb{R}^{n_{1} \times n_{2}}}\left\{\|X\|_{*}: \mathcal{A} X=\mathbf{b}\right\} .
\end{gathered}
$$

We know that the problems (1.3) and (1.4) are equivalent to (1.1) and (1.2), respectively, when the measure matrix $A$ and operator $\mathcal{A}$ satisfy some recovery guarantees, i.e., NSP and RIP conditions [14]. However, the solutions of (1.3) and (1.4) are usually suboptimal to the original problems (1.1) and (1.2), the $\ell_{1}$-norm minimization problem may yield the vector with lower sparse rate than the real one, in which a sparse target can't be recovered with minimum measurements [22]. Another limitation is its bias caused by shrinking all the elements toward zero simultaneously [21]. In addition, the nuclear norm of a matrix is the summation of its singular values, therefore it also has these limitations.

The obtained solution by $\ell_{1}$-minimization is usually suboptimal to the original $\ell_{0}$ minimization since the $\ell_{1}$-norm is a loose approximation of the $\ell_{0}$-norm [26]. In CS recovery problems, many well known nonconvex surrogates of $\ell_{0}$-norm have been proposed, including $\ell_{p}$-norm $(0<p<1)$ [15], Smoothly Clipped Absolute Deviation (SCAD) [13], Minimax Concave Penalty (MCP) [44], Exponential-Type Penalty (ETP) [17], Capped 
$\ell_{1}$ [45], Logarithm [16], Geman and Yang [18], Laplace [40] and some continuous $\ell_{0}$ functions [29]. For MC problems, some of the nonconvex methods also have been proposed recently $[23,25,35,22]$. The work of Nikolova, $\mathrm{Ng}$ and Tam [37] has shown that the nonconvex nonsmooth potential function (PF, including $\frac{\alpha|t|}{1+\alpha|t|}$ and $\log (\alpha|t|+1)$, e.t.c..) models enjoy better properties than the convex one in image restoration. Then, we want to extend it into CS and MC problems. What's more, to the best of our knowledge, there don't exist theoretical analyses of the recovery guarantees for these noncovex regu36 larized problems, except for $\ell_{p}$-norm $(0<p<1)$ and some continuous $\ell_{0}$ functions [29]. Therefore, it is significant to study the recovery guarantees for our proposed noncovex nonsmooth method.

In this paper, we will consider the following nonconvex nonsmooth PF regularized CS and MC problems:

$$
\begin{gathered}
\min _{\mathbf{x} \in \mathbb{R}^{p}}\{\Phi(\mathbf{x}): A \mathbf{x}=\mathbf{y}\}, \\
\min _{X \in \mathbb{R}^{n_{1} \times n_{2}}}\{\hat{\Phi}(X): \mathcal{A} X=\mathbf{b}\},
\end{gathered}
$$

where $\Phi(\mathbf{x})$ and $\hat{\Phi}(X)$ are defined by

$$
\Phi(\mathbf{x})=\sum_{i=1}^{p} \varphi\left(x_{i}\right), \hat{\Phi}(X)=\sum_{i=1}^{m} \varphi\left(\sigma_{i}(\mathrm{X})\right)
$$

where we choose PF as $\varphi(t)=\frac{\alpha|t|}{1+\alpha|t|}[11,33,42]$.

One can observe that $\Phi(\mathbf{x})$ convergent to $\|\mathbf{x}\|_{0}$, as $\alpha \rightarrow \infty$. It has been shown in [32] that $\varphi$ satisfies: (1) $\varphi$ is continuous (Lipschitz function), symmetric on $(-\infty, \infty), \mathcal{C}^{2}$ on $(0, \infty)$ and $\varphi(0)=0$ is a strict minimum; $(2) \varphi^{\prime}\left(0^{+}\right)>0$ and $\varphi^{\prime}(t) \geq 0$ for all $t>0$; (3) $\varphi^{\prime \prime}$ is increasing on $(0, \infty)$ with $\varphi^{\prime \prime}(t)<0$ and $\lim _{t \rightarrow \infty} \varphi^{\prime \prime}(t)=0$. These properties imply our regularizers to be good sparsity promoted penalty functions. Some other properties

48 of our regularizers are shown as follows:

(1) $\alpha>0, \hat{\Phi}(X) \geq 0(\Phi(\mathbf{x}) \geq 0)$, with equality holds if only if $X=\mathbf{0}(\mathbf{x}=\mathbf{0})$;

(2) $\hat{\Phi}(X)(\Phi(\mathbf{x}))$ is a decreasing function of $\alpha$, and $\lim _{\alpha \rightarrow \infty} \hat{\Phi}(X)=\operatorname{rank}(X)\left(\lim _{\alpha \rightarrow \infty} \Phi(\mathbf{x})=\right.$ $\left.\|\mathbf{x}\|_{0}\right)$;

(3) $\hat{\Phi}(X)$ is unitarily invariant, that is $\hat{\Phi}\left(U X V^{*}\right)=\hat{\Phi}(X)$ whenever $U \in \mathbb{R}^{n_{1} \times n_{1}}$ and $V \in \mathbb{R}^{n_{2} \times n_{2}}$ are orthogonal matrices.

The main contributions of this work include as follows. (1) We propose nonconvex nonsmooth PF regularized CS and MC problems. These are accomplished by adopting a 
nonconvex nonsmooth PF penalty that depends on the elements of vector $\mathbf{x}$ and singular values of matrix $X$, respectively. (2) Due to the nonconvex nonsmooth property of our proposed regularizer, minimizing (1.5) and (1.6) become challengeable. Thus, we propose an alternating minimization scheme in which the problem can be decomposed into three subproblems, two of them are convex and the remaining one is smooth. Therefore, some powerful optimization tools can be applied to deal with these three subproblems. (3) We prove that our alternating minimization algorithms converge to a critical point. (4) We also show the recovery guarantees for (1.5) and (1.6).

The rest of this paper is organized as follows. In Sect. 2, we focus on the nonconvex nonsmooth regularized models and present an alternating minimization algorithm. And we also analysis the convergence of the alternating algorithm. In Sect. 3, we show the recovery guarantees for our proposed methods, including NSP and RIP conditions. In Sect. 4, we give various numerical experiments about our proposed augmented models with simulated and real data. Finally, some conclusions are summarized in Sect. 5.

Notions and Preliminaries: We use $\mathbb{R}^{p}$ to denote the $p$-dimensional Euclidean space, $\langle\cdot, \cdot\rangle$ to denote the inner product, $\|\cdot\|$ to denote the norm induced from the inner product, $\mathbb{R}^{n_{1} \times n_{2}}$ to denote the $n_{1} \times n_{2}$-dimensional Euclidean space, and $\langle\cdot, \cdot\rangle_{\mathcal{X}}$ to denote the standard inner product, i.e., $\langle X, Y\rangle_{\mathcal{X}}=\operatorname{trace}\left(X^{T} Y\right), \forall X, Y \in \mathbb{R}^{n_{1} \times n_{2}}$, where $X^{T}$ denotes the transpose of $X$. For any $\mathbf{x} \in \mathbb{R}^{p}, \mathbf{x}_{\mathcal{S}}=\left(x_{\mathcal{S}[1]}, \ldots, x_{\mathcal{S}[\sharp \mathcal{S}]}\right) \in \mathbb{R}^{\sharp \mathcal{S}}$ and $\overline{\mathcal{S}}=\mathbb{I}_{p} / \mathcal{S}$, where $\sharp$ denotes cardinality and $\mathbb{I}_{p}=\{1, \ldots, p\}$. For any $\mathbf{x}=\left(x_{1}, \ldots, x_{p}\right) \in \mathbb{R}^{p}$ and $q>0,\|\cdot\|_{q}$ denotes the standard $\ell_{q}$-norm of $\mathbf{x}$ in $\mathbb{R}^{p}$, i.e., $\|\mathbf{x}\|_{q}=\left(\sum_{i=1}^{p}\left|x_{i}\right|^{q}\right)^{\frac{1}{q}}$. Particularly, $\|\cdot\|_{0}$ denotes the $\ell_{0}$-norm of $\mathbf{x}$, i.e., $\|\mathbf{x}\|_{0}=\sharp\left\{x_{i}, i=1, \ldots, p: x_{i} \neq 0\right\}$. Also, $\|\cdot\|_{*}$ denotes the nuclear-norm of $Y \in \mathbb{R}^{n_{1} \times n_{2}}$, i.e., $\|Y\|_{*}=\sum_{i=1}^{m} \sigma_{i}(Y)$, where $\sigma_{i}$ is the $i$ largest singular value of $Y$ and $m=\min \left\{n_{1}, n_{2}\right\}$, and $\|\cdot\|_{F}$ denotes Frobenius norm of $Y$, i.e, $\|Y\|_{F}^{2}=\sum_{i=1}^{m} \sigma_{i}^{2}(Y)$. We say that $u \in \mathcal{C}^{l}(\Omega)$ if it is $l$ times continuously differentiable functions with compact support. Let $\mathcal{X}, \mathcal{Y}$ be Hilbert spaces and $\mathcal{G} \subset \mathcal{X}$ be a dense linear subspace and $\mathcal{A}: \mathcal{G} \rightarrow \mathcal{Y}$ a linear operator. There exists a unique linear operator $\mathcal{A}^{*}: \operatorname{Dom}\left(\mathcal{A}^{*}\right) \subset \mathcal{Y} \rightarrow \mathcal{X}$ such that $\left\langle\mathcal{A}^{*} \mathbf{y}, \mathbf{x}\right\rangle=\langle\mathbf{y}, \mathcal{A} \mathbf{x}\rangle$, for any $\mathbf{x} \in \mathcal{G}$ and $\mathbf{y} \in \operatorname{Dom}\left(\mathcal{A}^{*}\right)$, where $\operatorname{Dom}\left(\mathcal{A}^{*}\right):=\{\mathbf{y} \in \mathcal{Y}: \mathbf{x} \rightarrow\langle\mathbf{y}, \mathcal{A} \mathbf{x}\rangle$ is continuous on $\mathcal{G}\}$. For any convex function $h$ and $\mathbf{x}, \mathbf{y} \in \mathbb{R}^{p}, \nabla h(\mathbf{x})(\partial h(\mathbf{x}))$ is the set of gradient (subgradient) at 
$\mathbf{x}$, in particular,

$$
\partial h(\mathbf{x})=\left\{\xi \in \mathbb{R}^{p}: h(\mathbf{y}) \geq h(\mathbf{x})+\langle\xi, \mathbf{y}-\mathbf{x}\rangle\right\} .
$$

For any function $g: \mathbb{R}^{n_{1} \times n_{2}} \rightarrow \mathbb{R}$ and $Y, \Delta Y \in \mathbb{R}^{n_{1} \times n_{2}}$, operator $D g(Y): \mathbb{R}^{n_{1} \times n_{2}} \rightarrow \mathbb{R}$ denotes the Gateaux derivatives at $Y$, in particular,

$$
\lim _{t \rightarrow 0} \frac{1}{t}|g(Y+t \Delta Y)-g(Y)-t\langle D g(Y), \Delta Y\rangle|=0 .
$$

One important property which we will use later on is the Kurdyka-Łojasiewicz (KL) property.

72 Definition 1.1. (KL function). A proper function $f: \mathbb{R}^{p} \rightarrow \mathbb{R}$ is said to have the Kurdyka-Lojasiewicz (KL) property at $\widehat{\mathbf{x}} \in \operatorname{Dom}(\partial f)\left(\equiv\left\{\mathbf{z} \in \mathbb{R}^{p}: \partial f(\mathbf{z}) \neq \varnothing\right\}\right)$ if there exist $\eta \in[0, \infty)$, a neighborhood $\mathcal{V}$ of $\widehat{\mathbf{x}}$, and a continuous concave function $\theta:[0, \eta) \rightarrow \mathbb{R}_{+}$ such that

- $\theta(0)=0$;

- $\theta$ is continuous differentiable on $(0, \eta)$;

- for all $z \in(0, \eta), \theta^{\prime}(z)>0$;

- for all $\mathbf{z} \in \mathcal{V} \cap[f(\widehat{\mathbf{z}})<f<f(\widehat{\mathbf{z}})+\eta]$, the Kurdyka-Eojasiewicz inequality and

$$
\theta^{\prime}(f(\mathbf{z})-f(\widehat{\mathbf{z}})) \operatorname{dist}(0, \partial f(\mathbf{z})) \geq 1
$$

hold, where $\operatorname{dist}(\mathbf{z}, \mathcal{U})=\inf \|\mathbf{y}-\mathbf{z}\|, \mathbf{y} \in \mathcal{U}$, for any $\mathcal{U} \subset \mathbb{R}^{p}$. Moreover, proper semicontinuous functions which satisfy the Kurdyka-Eojasiewicz inequality at each point of $\operatorname{Dom}(\partial f)$ are called KL functions.

Definition 1.2. (local Lipschitz condition). A function $f: \mathbb{R}^{p} \rightarrow \mathbb{R}$ is said to be local Lipschitz at $\mathbf{x}$ if

$$
\exists \varepsilon>0, \forall\left(\mathbf{y}, \mathbf{y}^{\prime}\right) \in \mathcal{B}(\mathbf{x}, \varepsilon)^{2}\left|f(\mathbf{y})-f\left(\mathbf{y}^{\prime}\right)\right| \leq L\left\|\mathbf{y}-\mathbf{y}^{\prime}\right\|,
$$

where $L$ is a nonnegative scalar and $\mathcal{B}(\mathbf{x}, \varepsilon)$ is a $\varepsilon$-neighborhood of $\mathbf{x}$.

Definition 1.3. (generalized gradient). The generalized gradient of a locally Lipschitz function $f: \mathbb{R}^{p} \rightarrow \mathbb{R}$ at $\mathbf{x}$, denoted $\bar{\partial} f(\mathbf{x})$, is defined by

$$
\bar{\partial} f(\mathbf{x}):=\left\{\xi \in \mathbb{R}^{p}: \bar{d} \geq\langle\mathbf{z}, \theta\rangle \forall \mathbf{z} \in \mathbb{R}^{p}\right\},
$$


where $\bar{d}(\mathbf{x}, \mathbf{z})$ denotes the generalized directional derivative of $f$ at $\mathbf{x}$ in the direction $\mathbf{z}$,

$$
\bar{d}(\mathbf{x}, \mathbf{z})=\limsup _{\mathbf{y} \rightarrow \mathbf{x}} \frac{f(\mathbf{y}+\eta \mathbf{z})-f(\mathbf{y})}{\eta} .
$$

If $f$ attains a local minimum or maximum at $\mathbf{x}$, then $\mathbf{0} \in \bar{\partial} f(\mathbf{x})$.

\section{${ }_{84}$ 2. Algorithm and Convergence Analysis}

In this section, we mainly propose an alternating minimization scheme for solving (1.5) and (1.6). We begin with introducing an auxiliary variable and then give the corresponding augmented Lagrangian function. And we use an alternating minimization scheme to solve this problems. Finally, we give the convergence analysis for our proposed method.

\subsection{Alternating Direction Method for Nonconvex Nonsmooth Regularized Problems}

In this subsection, we mainly focus on the MC, and it is fairly easy to extend the result to CS. Firstly, by introducing an auxiliary variable $W,(1.6)$ can be equivalently transformed into

$$
\min _{X, W \in \mathbb{R}^{n_{1} \times n_{2}}}\left\{\hat{\Psi}(X)+\alpha\|W\|_{*}: \mathcal{A} X=\mathbf{b}, X=W\right\},
$$

where $\hat{\Psi}(\mathrm{X})$ is Gateaux-differentiable at any bounded set of $\mathbb{R}^{n_{1} \times n_{2}}$.

Remark 1. In [32], we know that $\varphi^{\prime}(0)$ doesn't exist. From [41, 34], we know that, the

96 nonexistence of some partial derivatives $\frac{\partial \varphi\left(\sigma_{i}\right)}{\sigma_{i}}\left(\sigma_{i}=0\right.$ for $\left.i>r\right)$, imply that $\hat{\Phi}(\mathrm{X})$ is not Gateaux-differentiable.

Next, we consider the corresponding augmented Lagrangian function of (2.1):

$$
L_{\rho}(X, W, Z)=\frac{1}{2}\|\mathcal{A} X-\mathbf{b}\|^{2}+\lambda \hat{\Psi}(X)+\lambda \alpha\|W\|_{*}-\langle Z, W-X\rangle_{\mathcal{X}}+\frac{\rho}{2}\|W-X\|_{F}^{2},
$$

where $Z \in \mathbb{R}^{n_{1} \times n_{2}}$ is the Lagrangian multiplier, $\lambda$ is the regularization parameter and $\rho>0$ is the penalty parameter for the violation of the linear constraint.

Given $\left(X^{(s)}, W^{(s)}, Z^{(s)}\right)$, the iteration scheme of (2.2) can be described as follows:

$$
\left\{\begin{array}{c}
W^{(s+1)} \in \arg \min _{W \in \mathbb{R}^{n_{1} \times n_{2}}} L_{\rho}\left(X^{(s)}, W, Z^{(s)}\right) ; \\
X^{(s+1)} \in \arg \min _{X \in \mathbb{R}^{n_{1} \times n_{2}}} L_{\rho}\left(X, W^{(s+1)}, Z^{(s)}\right) ; \\
Z^{(s+1)}=Z^{(s)}-\rho\left(W^{(s+1)}-X^{(s+1)}\right) . \\
6
\end{array}\right.
$$


It's easy to know that the $W$-subproblem can be reformulated as

$$
\begin{aligned}
W^{(s+1)} & \in \arg \min _{W \in \mathbb{R}^{n_{1} \times n_{2}}} \frac{\rho}{2}\left\|X^{(s)}-W\right\|_{F}^{2}+\lambda \alpha\|W\|_{*}-\left\langle Z^{(s)}, W-X^{(s)}\right\rangle_{\mathcal{X}} \\
& =\arg \min _{W \in \mathbb{R}^{n_{1} \times n_{2}}} \frac{1}{2}\left\|W-\left(X^{(s)}+\frac{1}{\rho} Z^{(s)}\right)\right\|_{F}^{2}+\tau\|\mathrm{W}\|_{*},
\end{aligned}
$$

where $\tau=\frac{\lambda \alpha}{\rho}$. And it's also easy to know that the closed-form solution of (2.3) is

$$
W^{(s)}=\mathcal{D}_{\tau}\left(Y^{(s)}\right)
$$

where $Y^{(s)}=X^{(s)}+\frac{1}{\rho} Z^{(s)}, \mathcal{D}_{\tau}$ is the soft-thresholding operator, $\mathcal{D}_{\tau}=U \mathcal{D}_{\tau}(\Sigma) V^{*}$, $\mathcal{D}_{\tau}(\Sigma)=\operatorname{diag}\left(\left\{\sigma_{i}-\tau\right\}_{+}\right), t_{+}$is the positive part of $t$, namely, $t_{+}=\max (0, t)$ and $X=U \Sigma V^{*}$ is the singular value decomposition (SVD) of matrix $Y^{(s)}[3,46]$.

The $X$-subproblem can be formulated as follows

$$
X^{(s+1)}=\arg \min _{X \in \mathbb{R}^{n_{1} \times n_{2}}} \frac{1}{2}\|\mathcal{A} X-\mathbf{b}\|^{2}+\lambda \hat{\Psi}(X)+\frac{\rho}{2}\left\|W^{(s+1)}-X\right\|_{F}^{2}-\left\langle Z^{(s)}, W^{(s+1)}-X\right\rangle_{\mathcal{X}} .
$$

we can use Quasi-Newton's method to solve this optimization problem (2.4), we have

$$
\begin{gathered}
X^{(s+1)}=X^{(s)}+\Delta X \\
\left(\mathcal{A}^{*} \mathcal{A}+\rho \mathcal{I}\right) \Delta X=\mathcal{A}^{*}\left(\mathbf{b}-\mathcal{A} X^{(s)}\right)-\lambda D_{X} \hat{\Psi}\left(X^{(s)}\right)+\rho\left(W^{(s+1)}-X^{(s)}\right)-Z^{(s)},
\end{gathered}
$$

where $\mathcal{I}$ is the identity operator, and $\mathcal{A}^{*}$ is the adjoint of $\mathcal{A}$. What's more, it is easy to get $\Delta \mathrm{X}$ with the conjugate gradient method to solve the linear system (2.5).

Proposition 2.1. The Gateaux derivative of $\hat{\Psi}(X)$ is

$$
D_{X} \hat{\Psi}(X)=U \Lambda V^{*}
$$

where $\Lambda=\operatorname{diag}\left(\psi^{\prime}\left(\sigma_{1}\right), \ldots, \psi^{\prime}\left(\sigma_{m}\right)\right)_{n_{1} \times n_{2}}, \psi(t)=\varphi(t)-\alpha|t|$ and $U, V$ are unitary matrices which consist of the left-singular vectors and right-singular vectors.

The proof of proposition 2.1 is given in Appendix (A).

Based on the analysis above, we give a framework of the alternating minimization scheme for solving our proposed method of MC as follows: 


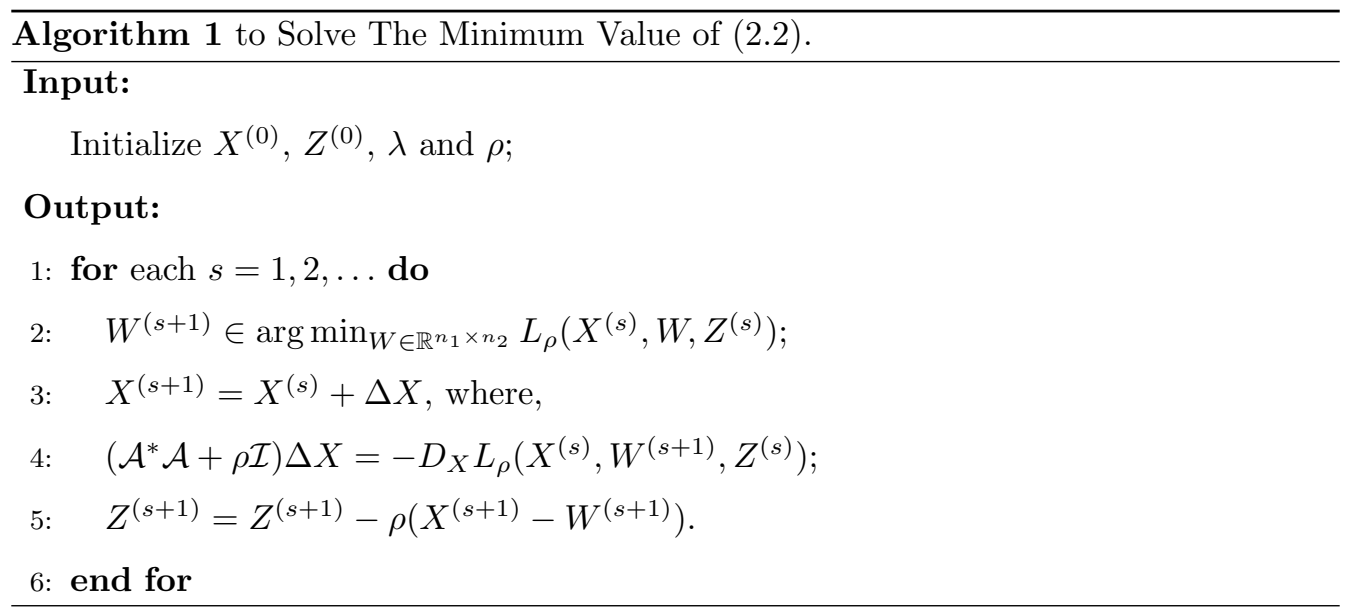

\subsection{Convergence Analysis}

The main aim of this section is to show that the Algorithm 1 converges to a critical point of (2.2). Now we can establish the properties of $\left(X^{(s)}, W^{(s)}, Z^{(s)}\right)$, and use them to show the convergence of Algorithm 1.

Proposition 2.2. Suppose that $\underline{\lambda}+\rho-\frac{L}{2}>0$, where $\underline{\lambda}=\lambda_{\min }\left(\mathcal{A}^{*} \mathcal{A}\right)$ and $L$ is the

Lipschitz constant of $D_{X} L_{\rho}\left(X, W^{(s+1)}, Z^{(s)}\right)$. Then, the sequences $\left(X^{(s)}, W^{(s)}, Z^{(s)}\right)$ generated by Algorithm 1 satisfy the following inequalities and equality:

(i)

$$
\begin{aligned}
\frac{\rho}{2}\left\|W^{(s+1)}-X^{(s)}\right\|_{F}^{2}+ & \lambda \alpha\left\|W^{(s+1)}\right\|_{*}-\left\langle Z^{(s)}, W^{(s+1)}-X^{(s)}\right\rangle_{\mathcal{X}}+\mu_{1}\left\|W^{(s)}-W^{(s+1)}\right\|_{F}^{2} \\
\leq & \frac{\rho}{2}\left\|W^{(s)}-X^{(s)}\right\|_{F}^{2}+\lambda \alpha\left\|W^{(s)}\right\|_{*}-\left\langle Z^{(s)}, W^{(s)}-X^{(s)}\right\rangle_{\mathcal{X}} .
\end{aligned}
$$

(ii)

$$
\begin{aligned}
& \frac{1}{2}\left\|\mathcal{A} X^{(s+1)}-\mathbf{b}\right\|^{2}+\lambda \hat{\Psi}\left(X^{(s+1)}\right)+\frac{\rho}{2}\left\|W^{(s+1)}-X^{(s+1)}\right\|_{F}^{2}-\left\langle Z^{(s)}, W^{(s+1)}-X^{(s+1)}\right\rangle_{\mathcal{X}} \\
& \quad+\mu_{2}\left\|X^{(s)}-X^{(s+1)}\right\|_{F}^{2} \\
& \quad \leq \frac{1}{2}\left\|\mathcal{A} X^{(s)}-\mathbf{b}\right\|^{2}+\lambda \hat{\Psi}\left(X^{(s)}\right)+\frac{\rho}{2}\left\|W^{(s+1)}-X^{(s)}\right\|_{F}^{2}-\left\langle Z^{(s)}, W^{(s+1)}-X^{(s)}\right\rangle_{\mathcal{X}}
\end{aligned}
$$

(iii)

$$
\left\langle Z^{(s)}-Z^{(s+1)}, W^{(s)}-X^{(s)}\right\rangle_{\mathcal{X}}=\rho\left\|Z^{(s)}-Z^{(s+1)}\right\|_{F}^{2} .
$$

The proof of proposition 2.2 is given in Appendix (B). 
Theorem 2.1. For any $T>0$, and the sequences $\left(X^{(s)}, W^{(s)}, Z^{(s)}\right)$ generated by Algorithm 1 , there exist a constant $\kappa>0$ satisfy the following inequality

$$
\sum_{s=1}^{T} \operatorname{dist}\left(\partial L_{\rho}\left(X^{(s)}, W^{(s)}, Z^{(s)}\right), \boldsymbol{O}\right)^{2} \leq \frac{1}{\kappa}\left[L_{\rho}\left(X^{(0)}, W^{(0)}, Z^{(0)}\right)-L_{\rho}\left(X^{(T)}, W^{(T)}, Z^{(T)}\right)\right] .
$$

The proof of theorem 2.1 is given in Appendix (C).

Remark 2. In literature [19], we could use $\operatorname{dist}\left(\partial L_{\rho}\left(X^{(s)}, W^{(s)}, Z^{(s)}\right), \mathbf{0}\right)^{2} \leq \epsilon$ to judge approximate stationarity of $\left(X^{(s)}, W^{(s)}, Z^{(s)}\right)$. Contrast this with convex optimization, where one uses $\left[L_{\rho}\left(X^{(s)}, W^{(s)}, Z^{(s)}\right)-L_{\rho}\left(X^{*}, W^{*}, Z^{*}\right)\right]$ or $\left\|X-X^{*}\right\|_{F}^{2}+\left\|W-W^{*}\right\|_{F}^{2}+$ ${ }_{132}\left\|Z-Z^{*}\right\|_{F}^{2}$ as criteria for convergence analysis.

Remark 3. From (2.9), we know that our algorithm finds a $\epsilon$-accurate solution in time $o\left(\frac{1}{\epsilon^{2}}\right)$.

According to the definition of $\hat{\Psi}$ we know that it is a KL function. Our objective is to show that the sequence which is generated by Algorithm 1 converges to a critical point of (2.2). For this purpose we use the KL property to analyze the convergence.

Theorem 2.2. Let $\left(X^{(s)}, W^{(s)}, Z^{(s)}\right)$ be a sequence generated by Algorithm 1 with an initial point $\left(X^{(0)}, W^{(0)}, Z^{(0)}\right)$. If $L_{\rho}$ has the Kurdyka-Eojasiewicz (KL) property at a critical point $\left(X^{*}, W^{*}, Z^{*}\right)$ with

$$
L_{\rho}\left(X^{*}, W^{*}, Z^{*}\right) \leq L_{\rho}\left(X^{(s)}, W^{(s)}, Z^{(s)}\right) \leq L_{\rho}\left(X^{*}, W^{*}, Z^{*}\right)+\eta
$$

and

$$
\begin{aligned}
C & +2 \sqrt{\frac{L_{\rho}\left(X^{(0)}, W^{(0)}, Z^{(0)}\right)-L_{\rho}\left(X^{*}, W^{*}, Z^{*}\right)}{a}} \\
& +\frac{b}{a} \theta\left(L_{\rho}\left(X^{(0)}, W^{(0)}, Z^{(0)}\right)-L_{\rho}\left(X^{*}, W^{*}, Z^{*}\right)\right)<\varrho .
\end{aligned}
$$

where $C=\left\|X^{(0)}-X^{*}\right\|_{F}^{2}+\left\|W^{(0)}-W^{*}\right\|_{F}^{2}+\left\|Z^{(0)}-Z^{*}\right\|_{F}^{2}, a=\min \left\{\mu_{1}, \mu_{2}, \rho\right\}, b=$ $\lambda L^{\prime}+\rho+\frac{1}{\rho}+2$ and $D \hat{\Psi}$ is a $L^{\prime}$-Lipschitz function. Then, we have

$$
\sum_{s=0}^{+\infty}\left[\left\|X^{(s+1)}-X^{(s+1)}\right\|_{F}^{2}+\left\|W^{(s+1)}-W^{(s)}\right\|_{F}^{2}+\left\|Z^{(s+1)}-Z^{(s)}\right\|_{F}^{2}\right]^{\frac{1}{2}} \leq+\infty,
$$

and

$$
\lim _{s \rightarrow+\infty}\left(X^{(s)}, W^{(s)}, Z^{(s)}\right)=\left(X^{*}, W^{*}, Z^{*}\right) .
$$

The proof of theorem 2.2 is given in Appendix (D). 


\section{Recovery Guarantees}

In this section, we establish exact CS and MC recovery guarantees for our proposed models (1.5) and (1.6). The exact recovery guarantees are given based on the varieties of properties of $\mathrm{A}$ and $\mathcal{A}$ including NSP and RIP. These theoretical analyses ensure the effectiveness of the algorithms presented in Sect. 2. A note should be indicated here that the experiments in Sect. 4 are not all required to satisfy these recovery guarantees, because, in some sense, the local minimum is enough.

\subsection{1-D Case}

Definition 3.1. A matrix $A \in \mathbb{R}^{n \times p}$ satisfies the null-space property relative to a set $\mathcal{S} \subset[p]$ if

$$
\left\|\mathbf{h}_{\mathcal{S}}\right\|_{1} \leq\left\|\mathbf{h}_{\mathcal{S}}\right\|_{1}
$$

for all $\mathbf{h} \in \mathrm{NULL}(A) /\{0\}[20,12]$.

It is said to satisfy the null-space property of order $k$ if it satisfies the null-space property related to any set $\mathcal{S} \subset[p]$ with $\operatorname{card}(\mathcal{S}) \leq k$. Given any vector $\mathbf{x} \in \mathbb{R}^{p}$ supported on a set $\mathcal{S}$ is the unique solution of (1.3) if and only if $A$ satisfies the null-space property relative to $\mathcal{S}$. Next, we extend the NSP condition to our augment model (1.5).

Theorem 3.1. (NSP condition for exact recovery). The problem (1.5) uniquely recovers $k$-sparse vector $\mathbf{x}_{0}$ from measurement $A \mathbf{x}_{0}=\mathbf{y}$ if

$$
2\left\|\mathbf{h}_{\mathcal{S}}\right\|_{1} \leq\left\|\mathbf{h}_{\mathcal{S}}\right\|_{1}
$$

holds for all vectors $\mathbf{h} \in N U L L(A)$ and coordinate sets $\mathcal{S}$ with cardinality $|\mathcal{S}| \leq k$.

ProOF.

$$
\begin{aligned}
& \Phi\left(\mathbf{x}_{0}+\mathbf{h}\right)=\alpha\left\|\mathbf{x}_{0}+\mathbf{h}\right\|_{1}+\Psi\left(\mathbf{x}_{0}+\mathbf{h}\right) \\
& =\alpha\left\|\mathbf{x}_{0}+\mathbf{h}_{\mathcal{S}}\right\|_{1}+\Psi\left(\mathbf{x}_{0}+\mathbf{h}_{\mathcal{S}}\right)+\alpha\left\|\mathbf{h}_{\overline{\mathcal{S}}}\right\|_{1}+\Psi\left(\mathbf{h}_{\overline{\mathcal{S}}}\right) \\
& \geq \alpha\left\|\mathbf{x}_{0}\right\|_{1}-\alpha\left\|\mathbf{h}_{\mathcal{S}}\right\|_{1}+\Psi\left(\mathbf{x}_{0}\right)+\Psi\left(\mathbf{x}_{0}+\mathbf{h}_{\mathcal{S}}\right)-\Psi\left(\mathbf{x}_{0}\right)+\alpha\left\|\mathbf{h}_{\overline{\mathcal{S}}}\right\|_{1}+\Psi\left(\mathbf{h}_{\overline{\mathcal{S}}}\right) \\
& \geq\left[\alpha\left\|\mathbf{x}_{0}\right\|_{1}+\Psi\left(\mathbf{x}_{0}\right)\right]-\alpha\left\|\mathbf{h}_{\mathcal{S}}\right\|_{1}+\Psi\left(\mathbf{x}_{0}+\mathbf{h}_{\mathcal{S}}\right)-\Psi\left(\mathbf{x}_{0}\right)+\alpha\left\|\mathbf{h}_{\overline{\mathcal{S}}}\right\|_{1},
\end{aligned}
$$


where the first inequality from the triangle inequality and the second follows from $\Psi\left(\mathbf{h}_{\overline{\mathcal{S}}}\right)>0$. And we have

$$
\begin{aligned}
\Psi\left(\mathbf{x}_{0}+\mathbf{h}_{\mathcal{S}}\right) & -\Psi\left(\mathbf{x}_{0}\right) \\
& =\sum_{i \in \mathcal{S}} \frac{\alpha^{2}\left[x_{i}^{2}+\alpha x_{i}^{2}\left|x_{i}+h_{i}\right|-\left(x_{i}+h_{i}\right)^{2}-\alpha\left|x_{i}\right|\left(x_{i}+h_{i}\right)^{2}\right]}{\left(1+\alpha\left|x_{i}\right|\right)\left(1+\alpha\left|x_{i}+h_{i}\right|\right)} \\
& \geq \sum_{i \in \mathcal{S}} \frac{\alpha^{2}\left[\alpha\left|x_{i}\right| \cdot\left|x_{i}+h_{i}\right| \cdot\left(\left|x_{i}\right|-\left|x_{i}+h_{i}\right|\right)-\left(2 x_{i}+h_{i}\right) h_{i}\right]}{\left(1+\alpha\left|x_{i}\right|\right)\left(1+\alpha\left|x_{i}+h_{i}\right|\right)} \\
& \geq \sum_{i \in \mathcal{S}}\left[-\frac{\alpha^{2}\left(1+\alpha\left|x_{i}\right|\right) \cdot\left|x_{i}+h_{i}\right| \cdot\left|h_{i}\right|}{\left(1+\alpha\left|x_{i}\right|\right)\left(1+\alpha\left|x_{i}+h_{i}\right|\right)}-\frac{\alpha^{2}\left|x_{i}\right| \cdot\left|h_{i}\right|}{\alpha\left|x_{i}\right| \cdot\left(1+\alpha\left|x_{i}+h_{i}\right|\right)}\right] \\
& =-\sum_{i \in \mathcal{S}} \alpha\left|h_{i}\right|=-\alpha\left\|\mathbf{h}_{\mathcal{S}}\right\|_{1},
\end{aligned}
$$

where we made use of the relation $|a+b| \geq|a|-|b|$ for the second inequality. Combining with (3.2) and (3.3) we know that $\Phi\left(\mathbf{x}_{0}+\mathbf{h}\right)$ is strictly larger than $\Phi\left(\mathbf{x}_{0}\right)$ when (3.1) holds.

The RIP is a simple and useful measure for the quality of the measurement matrix $A$. It ensures the success of the sparse recovery algorithm presented in this paper. Next, we first review the RIP-based CS guarantees and then show that, given certain RIP conditions, guarantees exact recovery by (1.5).

Definition 3.2. The kth restricted isometry constant $\delta_{k}=\delta_{k}(A)$ of matrix $A \in \mathbb{R}^{n \times p}$ is the smallest $\delta \geq 0$ such that

$$
(1-\delta)\|\mathbf{x}\|_{2}^{2} \leq\|A \mathbf{x}\|_{2}^{2} \leq(1+\delta)\|\mathbf{x}\|_{2}^{2},
$$

for all $k$-sparse vectors $\mathbf{x} \in \mathbb{R}^{p}[\eta]$.

We say that $A$ satisfies the RIP if $\delta_{k}$ is small for reasonably large $k$, then we establish the success of sparse recovery via our proposed model (1.5) for measurement matrices with small restricted isometry constants.

${ }_{168}$ Theorem 3.2. (RIP condition for exact recovery). Assume that $\mathbf{x}_{0} \in \mathbb{R}^{n}$ is $k$-sparse. If A satisfies RIP with $\delta_{2 k} \leq 0.1273$, then $\mathbf{x}_{0}$ is the unique minimizer of (1.5) given by measurement $A \mathbf{x}_{0}=\mathbf{y}$. 
Proof. [30] shows that any vectors $\mathbf{h} \in \operatorname{NULL}(A)$ satisfies

$$
\left\|\mathbf{h}_{\mathcal{S}}\right\|_{1} \leq \theta_{2 k}\left\|\mathbf{h}_{\overline{\mathcal{S}}}\right\|_{1}
$$

where

$$
\theta_{2 k}:=\sqrt{\frac{4\left(1+5 \delta_{2 k}-4 \delta_{2 k}^{2}\right)}{\left(1-\delta_{2 k}\right)\left(32-25 \delta_{2 k}\right)}}
$$

Hence, (3.1) holds, on condition that

$$
\theta_{2 k} \leq \frac{1}{2}
$$

in light of $\delta_{2 k}<1$, for $\delta_{2 k}=0.1273$, we obtain $\theta_{2 k} \approx \frac{1}{2}$.

\subsection{2-D Case}

It's easy to extend the NSP and RIP condition to MC. First, let us introduce some definitions and properties. Let $X$ and $W$ be two matrices of the same size, we have $\sum_{i=1}^{r} \varphi\left(\sigma_{i}(X)-\sigma_{i}(W)\right) \leq \hat{\Phi}(X-W)$, because $\left|\sigma_{i}(X)-\sigma_{i}(W)\right| \leq\left|\sigma_{i}(X-W)\right|$, for $i=1, \ldots, r$ and $\varphi$ is an increasing function.

Theorem 3.3. (NSP condition for exact matrix completion). Problem (1.6) uniquely recovers all matrices $X_{0}$ of rank $r$ or less from measurement $\mathcal{A} X_{0}=\mathbf{b}$ if

$$
2 \sum_{i=1}^{r} \sigma_{i}(H) \leq \sum_{i=r+1}^{m} \sigma_{i}(H)
$$

holds for all matrices $H \in \operatorname{NULL}(\mathcal{A})$.

Proof.

$$
\begin{aligned}
\alpha\|X+H\|_{*}+\hat{\Psi}(X+H) \geq & \alpha \sum_{i=1}^{m}\left(\sigma_{i}(X)+\sigma_{i}(H)\right)+\sum_{i=1}^{m} \psi\left(\sigma_{i}(X)+\sigma_{i}(H)\right) \\
\geq & \alpha \sum_{i=1}^{r} \sigma_{i}(X)-\alpha \sum_{i=1}^{r} \sigma_{i}(H)+\alpha \sum_{i=r+1}^{m} \sigma_{i}(H) \\
& +\sum_{i=1}^{r} \psi\left(\sigma_{i}(X)+\sigma_{i}(H)\right)+\sum_{i=1+r}^{m} \psi\left(\sigma_{i}(H)\right) \\
\geq & {\left[\alpha \sum_{i=r+1}^{m} \sigma_{i}(H)-2 \alpha \sum_{i=1}^{r} \sigma_{i}(H)\right]+\sum_{i=1+r}^{m} \psi\left(\sigma_{i}(H)\right) } \\
& +\left[\alpha\|X\|_{*}+\hat{\Psi}(X)\right] .
\end{aligned}
$$


The NSP condition on the operator $\mathcal{A}$ ensures exact reconstruction of the original lowrank matrix $X_{0}$ in (1.6) even though the penalty term is nonconvex.

The RIP is also a simple and useful measure for the quality of operator $\mathcal{A}$. It ensures the success of the MC algorithm presented in this paper. Similar to analysis of CS above, we first review the RIP-based MC guarantees and then show that, given certain RIP conditions, guarantees exact MC by (1.6).

Definition 3.3. For a linear map $\mathcal{A}: \mathbb{R}^{n_{1} \times n_{2}} \rightarrow \mathbb{R}^{n_{3}}$ and for $r \leq m=\min \left\{n_{1}, n_{2}\right\}$, the rank restricted isometry constant $\delta_{r}=\delta_{r}(\mathcal{A})$ is the defined as the smallest $\delta \geq 0$ such that

$$
(1-\delta)\|X\|_{F}^{2} \leq\|\mathcal{A} X\|_{2}^{2} \leq(1+\delta)\|X\|_{F}^{2}
$$

for all matrices $X \in \mathbb{R}^{n_{1} \times n_{2}}$ of rank at most $r$ [36].

Theorem 3.4. (RIP condition for exact matrix completion). Let $X_{0}$ be a matrix with rank $r$ or less, the proposed model (1.6) exactly recovers $X_{0}$ from measurement $\mathbf{b}=\mathcal{A} X_{0}$ if $\mathcal{A}$ satisfies the $R I P$ condition with $\delta_{2 r} \leq 0.1273$.

Proof. In [24], it is established that every $H \in \operatorname{NULL}(\mathcal{A})$ satisfies $\sum_{i=1}^{r} \sigma_{i}(H) \leq$ $\theta_{2 r} \sum_{i=r+1}^{m} \sigma_{i}(H)$, hence (3.4) holds when $\theta_{2 r} \leq \frac{1}{2}$.

\section{Numerical Experiments}

\subsection{Parameters Settings}

Algorithm 1 is designed for solving (2.2) with a fixed regularization parameter $\lambda$. However, a good regularization parameter that leads to good approximation to the low rank target may not be known in advance. Similar to [22], we propose a data driven regularization parameter selection rules with discrepancy principle (DP). To precise, given a decreasing sequence of parameter $\left\{\lambda_{s}\right\}$, we run the Algorithm 1 to solve the $\lambda_{s+1}$-loop initialized with the solution of $\lambda_{s}$-loop. Meanwhile the discrepancy $d^{s}=$ $\left\|\mathcal{A} X\left(\lambda_{s}\right)-\mathbf{b}\right\|$ is calculated and stored at $\lambda_{s}$. The regularization parameter is selected as minimum that makes $d^{s}>\delta$, where $\delta$ is the noise level (see Algorithm 2 for detail). 


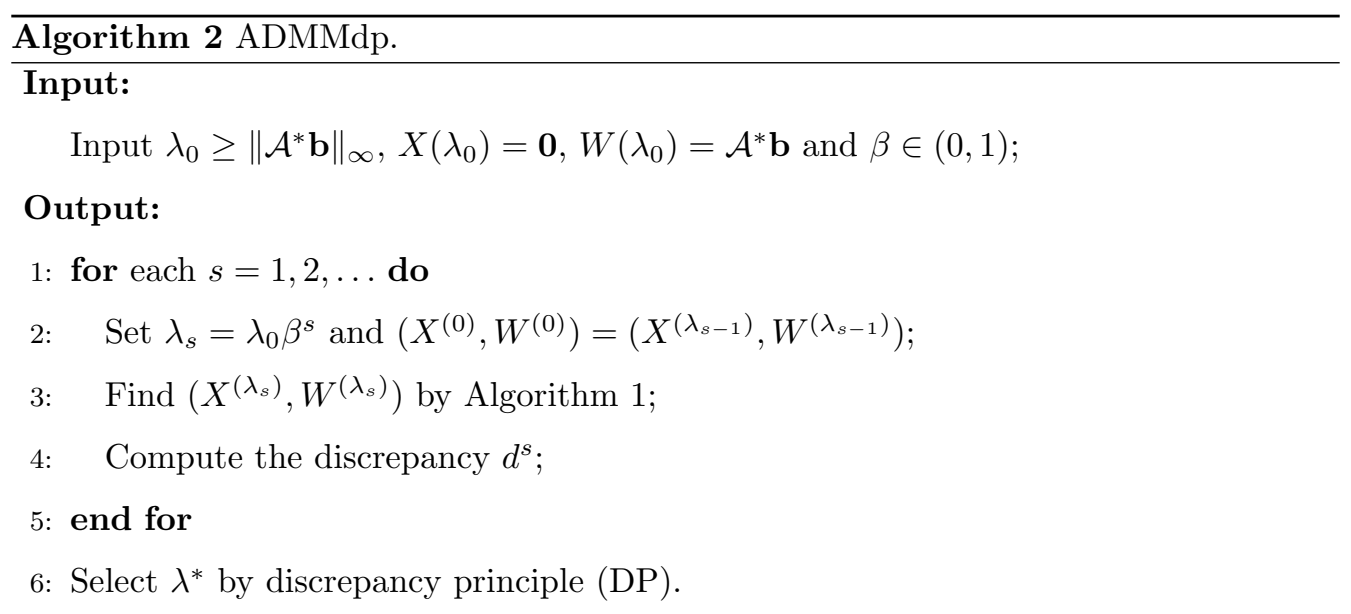

\subsection{Test on Compressed Sensing}

In this subsection, $n, m$ are the size of measure matrix $A$ and $s$ is the support size of original vector $\mathbf{x}$. We apply the proposed ADMMdp for solving the compressed sensing problem (1.5). In order to illustrate the performance of ADMMdp for nonconvex penalty compressed sensing problem, we compare the ADMMdp with the linearized Bregman iterations (LBI) method [2] and the MCP [44] method. And all the experiments in this section are performed under Window 10 and MATLAB 2013a running on a Lenovo laptop with an Intel(R) Core(TM) i5-3337U CPU at 1.8GHz and 4 GB memory. The LBI method solves the problem (1.3), the MCP and Capped- $\ell_{1}$ solves the following problem

$$
\min _{x \in \mathbb{R}^{p}}\left\{\frac{1}{2}\|A \mathbf{z}-\mathbf{y}\|^{2}+\sum_{i=1}^{p} \rho_{\lambda, \tau}\left(x_{i}\right): \mathbf{x}=\mathbf{z}\right\},
$$

where

$$
\rho_{\lambda, \tau}\left(x_{i}\right)= \begin{cases}\lambda\left(\left|x_{i}\right|-\frac{\left|x_{i}\right|^{2}}{2 \lambda \tau}\right) & \text { if }\left|x_{i}\right|<\tau \lambda, \\ \frac{\lambda^{2} \tau}{2} & \text { if }\left|x_{i}\right| \geq \tau \lambda .\end{cases}
$$

The corresponding augmeted Lagrangian function of (4.1) is

$$
L_{\rho}(\mathbf{x}, \mathbf{z}, \mathbf{p})=\frac{1}{2}\|A \mathbf{z}-\mathbf{y}\|^{2}+\sum_{i=1}^{p} \rho_{\lambda, \tau}\left(x_{i}\right)-\langle\mathbf{p}, \mathbf{x}-\mathbf{z}\rangle+\frac{\rho}{2}\|\mathbf{x}-\mathbf{z}\|^{2},
$$

where $\mathbf{p} \in \mathbb{R}^{p}$ is the Lagrangian multiplier, and $\rho>0$ is the penalty parameter for the violation of the linear constraint. 
Table 1: Nonconvex penalty functions $\rho_{\lambda, \tau}(t)$ and the thresholding operators $\mathcal{S}_{\lambda, \tau}^{\rho}(t)$.

\begin{tabular}{|c|c|c|c|c|}
\hline Penalty functions & $\rho_{\lambda, \tau}(t)$ & \multicolumn{3}{|c|}{$\mathcal{S}_{\lambda, \tau}^{\rho}(t)$} \\
\hline Lasso $[38]$ & $\lambda|t|$ & \multicolumn{3}{|c|}{$\operatorname{sign}(t) \max (|t|-\lambda, 0)$} \\
\hline Capped- $\ell_{1}[45]$ & $\begin{array}{ll}\lambda^{2} \tau & |t|>\lambda \tau \\
\lambda|t| & |t| \leq \lambda \tau\end{array}$ & & $\begin{array}{l}0 \\
\operatorname{sign}(t)(|t|-\lambda) \quad \lambda \leq \\
\operatorname{sign}(t)\left(|t| \pm \frac{\lambda}{2}\right) \\
t\end{array}$ & $\begin{array}{r}|t| \leq \lambda \\
|t|<\lambda\left(\tau+\frac{1}{2}\right) \\
|t|=\lambda\left(\tau+\frac{1}{2}\right) \\
|t| \geq \lambda\left(\tau+\frac{1}{2}\right)\end{array}$ \\
\hline $\operatorname{MCP}[43]$ & $\begin{cases}\lambda\left(|t|-\frac{t^{2}}{2 \lambda \tau}\right) & \mid t \\
\frac{\lambda^{2} \tau}{2} & \mid t\end{cases}$ & $\begin{array}{l}<\tau \lambda \\
\geq \tau \lambda\end{array}$ & $\operatorname{sign}(t) \frac{\tau(|t|-\lambda)}{\tau-1} \lambda$ & $\begin{array}{r}\quad|t|<\lambda \\
\leq|t|<\lambda \tau \\
\quad|t| \geq \lambda \tau\end{array}$ \\
\hline SCAD [13] & $\left\{\begin{array}{l}\frac{\lambda^{2}(\tau+1)}{2} \\
\frac{\lambda \tau|t|-\frac{1}{2}\left(t^{2}+\lambda^{2}\right)}{\tau-1} \\
\lambda|t|\end{array}\right.$ & $\begin{array}{r}|t|>\tau \lambda \\
\lambda<|t| \leq \tau \lambda \\
|t| \leq \lambda\end{array}$ & $\begin{array}{l}0 \\
\operatorname{sign}(t)(|t|-\lambda) \\
\operatorname{sign}(t) \frac{(\tau-1)|t|-\lambda}{\tau-2} \\
t\end{array}$ & $\begin{array}{r}|t| \leq \lambda \\
\lambda<|t| \leq 2 \lambda \\
-2 \lambda<|t| \leq \lambda \tau \\
|t|>\lambda \tau\end{array}$ \\
\hline
\end{tabular}

Given $\left(\mathbf{x}^{(s)}, \mathbf{z}^{(s)}, \mathbf{p}^{(s)}\right)$, the iteration scheme of (4.3) can be described as follows:

$$
\left\{\begin{array}{l}
\mathbf{x}^{(s+1)} \in \arg \min _{\mathbf{x} \in \mathbb{R}^{p}} L_{\rho}\left(\mathbf{x}, \mathbf{z}^{(s)}, \mathbf{p}^{(s)}\right) ; \\
\mathbf{z}^{(s+1)} \in \arg \min _{\mathbf{z} \in \mathbb{R}^{p}} L_{\rho}\left(\mathbf{x}^{(s+1)}, \mathbf{z}, \mathbf{p}^{(s)}\right) ; \\
\mathbf{p}^{(s+1)}=\mathbf{p}^{(s)}-\rho\left(\mathbf{x}^{(s+1)}-\mathbf{z}^{(s+1)}\right) .
\end{array}\right.
$$

The $\mathbf{x}$-subproblem can be reformulated as

$$
\mathbf{x}^{(s+1)} \in \arg \min _{\mathbf{x} \in \mathbb{R}^{p}} \sum_{i=1}^{p} \rho_{\lambda, \tau}\left(x_{i}\right)+\frac{\rho}{2}\left\|\mathbf{x}-\widehat{\mathbf{x}}^{(s)}\right\|^{2},
$$

where $\widehat{\mathbf{x}}^{(s)}=\mathbf{z}^{(s)}+\frac{\mathbf{p}^{(s)}}{\rho}$. The solution of (4.4) can be formulated as

$$
x_{i}^{(s+1)}=\mathcal{S}_{\lambda, \tau}^{\rho}\left(\widehat{\mathbf{x}}_{i}^{(s)}\right),
$$

where (4.5) is listed in Table 1 and the proofs of $\mathcal{S}_{\lambda, \tau}^{\rho}$ are given in Appendix (E). And the $\mathbf{z}$-subproblem can be solved by using the conjugate gradient method.

In this subsection, we test ADMMdp, MCP, Capped- $\ell_{1}$ and LBI for Gaussian measure matrix, Bernoulli measure matrix and partial DCT measure matrix. What's more, we use

$$
\left\|A \mathbf{x}^{(s)}-\mathbf{y}\right\| /\|\mathbf{y}\|<10^{-4}
$$


and the maximum iteration step maxit $=200$ as stopping criterion. And the relative error is defined as

$$
\text { RelErr }=\frac{\left\|\mathbf{x}^{o p t}-\mathbf{x}\right\|}{\|\mathbf{x}\|},
$$

where $\mathbf{x}^{\text {opt }}$ is outputted by the algorithm. The numerical results are reported in Table 2. From the Table 2, it can be seen that ADMMdp achieves much better recovery performance than the LBI. This is because the nonconvex penalty functions approximate the $\ell_{0}$ function better than the convex $\ell_{1}$ norm. From Fig. 1 , we know that ADMMdp can find the stationary point faster than MCP. We think that it is thanks to the convexity or smoothness of all the subproblems in Algorithm 2, its strict theoretical proof remains to be studied in the future work.

Table 2: Numerical result of ADMMdp, MCP, Capped- $\ell_{1}$ and LBI for compressed sensing.

\begin{tabular}{|c|c|c|c|c|c|c|c|c|c|c|c|c|}
\hline \multirow[t]{2}{*}{$(n, p, s)$} & \multicolumn{3}{|c|}{ ADMMdp } & \multicolumn{3}{|c|}{$\mathrm{MCP}$} & \multicolumn{3}{|c|}{ Capped- $\ell_{1}$} & \multicolumn{3}{|c|}{ LBI } \\
\hline & Time(s) & RelErr & $\sharp$ Iter & Time(s) & RelErr & $\sharp$ Iter & Time(s) & RelErr & $\sharp$ Iter & Time(s) & RelErr & $\sharp$ Iter \\
\hline & \multicolumn{12}{|c|}{ Gaussian matrices } \\
\hline$(250,500,25)$ & $1.07 \mathrm{e}+01$ & $1.68 \mathrm{e}-04$ & 145 & $1.57 \mathrm{e}+01$ & $6.49 \mathrm{e}-04$ & 200 & $2.30 \mathrm{e}+01$ & $3.55 \mathrm{e}-04$ & 200 & $1.09 \mathrm{e}+01$ & $3.48 \mathrm{e}-04$ & 200 \\
\hline \multirow[t]{2}{*}{$(500,1000,50)$} & $4.44 \mathrm{e}+01$ & $1.25 \mathrm{e}-04$ & 58 & $1.46 \mathrm{e}+02$ & $2.39 \mathrm{e}-04$ & 182 & $2.82 \mathrm{e}+01$ & $2.71 \mathrm{e}-04$ & 200 & $9.34 \mathrm{e}+01$ & $3.02 \mathrm{e}-04$ & 200 \\
\hline & \multicolumn{12}{|c|}{ Bernoulli matrices } \\
\hline$(250,500,25)$ & $1.42 \mathrm{e}+01$ & $2.01 \mathrm{e}-04$ & 200 & $1.67 \mathrm{e}+01$ & $8.77 \mathrm{e}-04$ & 200 & $2.74 \mathrm{e}+01$ & $9.64 \mathrm{e}-04$ & 200 & $1.16 \mathrm{e}+01$ & $3.57 \mathrm{e}-04$ & 200 \\
\hline \multirow[t]{2}{*}{$(500,1000,50)$} & $4.94 \mathrm{e}+01$ & $2.05 \mathrm{e}-04$ & 65 & $1.56 \mathrm{e}+02$ & $2.17 \mathrm{e}-04$ & 200 & $2.06 \mathrm{e}+02$ & $3.02 \mathrm{e}-04$ & 200 & $9.51 \mathrm{e}+01$ & $2.61 \mathrm{e}-04$ & 200 \\
\hline & \multicolumn{12}{|c|}{ Partial DCT matrices } \\
\hline$(250,500,25)$ & $3.42 \mathrm{e}+00$ & $2.77 \mathrm{e}-03$ & 200 & $3.48 \mathrm{e}+00$ & $4.30 \mathrm{e}-03$ & 200 & $5.53 \mathrm{e}+00$ & $4.23 \mathrm{e}-03$ & 200 & $2.87 \mathrm{e}+00$ & $5.19 \mathrm{e}-03$ & 200 \\
\hline$(500,1000,50)$ & $2.27 \mathrm{e}+01$ & $3.00 \mathrm{e}-03$ & 200 & $2.19 \mathrm{e}+01$ & $5.95 \mathrm{e}-03$ & 200 & $2.37 \mathrm{e}+01$ & $6.27 \mathrm{e}-03$ & 200 & $1.79 \mathrm{e}+01$ & $4.42 \mathrm{e}-03$ & 200 \\
\hline
\end{tabular}

\subsection{Test on Matrix Completion}

In this numerical experiments, $n_{1}$ and $n_{2}$ represent the matrix dimension, $r$ is the rank of original matrix, and $n_{3}$ denotes the number of measurement. Given $r \leq \min \left(n_{1}, n_{2}\right)$, we generate $\mathrm{X}=\mathrm{X}_{\mathrm{L}} \mathrm{X}_{\mathrm{R}}^{*}$, where matrices $\mathrm{X}_{\mathrm{L}} \in \mathbb{R}^{n_{1} \times r}$ and $\mathrm{X}_{\mathrm{R}} \in \mathbb{R}^{n_{2} \times r}$ are generated by independent identically distributed Gaussian entries. The subset $\Omega$ of $n_{3}$ elements is selected uniformly at random entries from $\left\{(i, j): i=1, \ldots, n_{1}, j=1, \ldots, n_{2}\right\}$ [22]. The linear measurement $\mathbf{b}$ is set to be $\mathbf{b}=\mathcal{A}(X)+\mathbf{n}$, where $\mathbf{n}$ is the additive Gaussian noise of zero mean and standard deviation $\sigma$, which will be specified in different test data sets. We use $s r=n_{3} /\left(n_{1} n_{2}\right)$ to denote the sampling ratio, and $d r=r\left(n_{1}+n_{2}-r\right)$ to denote 


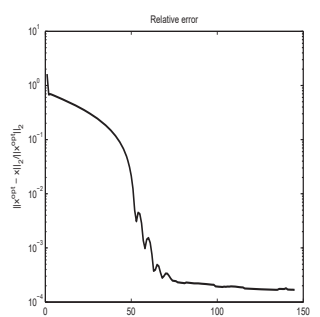

(a)

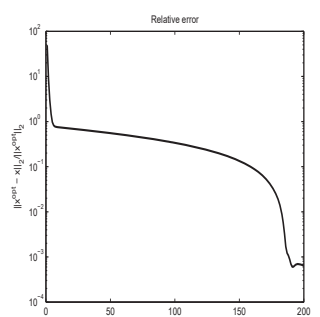

(b)

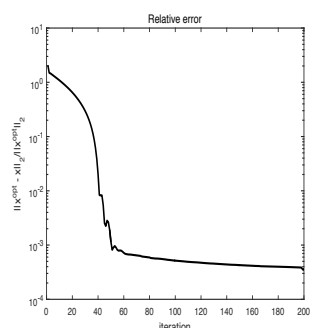

(c)

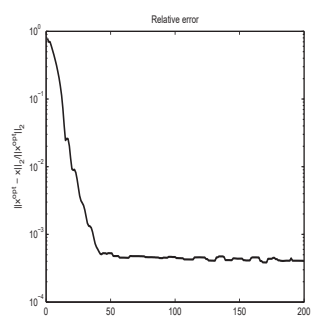

(d)

Figure 1: Relative error values of (a) recovered vector by ADMMdp (b) recovered vector by MCP (c) recovered vector by Capped- $\ell_{1}(\mathrm{~d})$ recovered vector by LBI with $n=250, n=500, n=25$ and Gaussian matrix $A$.

the number of degree of freedom for a real-valued rank $r$ matrix. As mentioned in [28], when the ratio $n_{3} / d r$ is greater than 3 , the problem can be viewed as an easy problem. On the contrary, it is called a hard problem. Another ratio is $F R>1$, it is impossible to recover matrix because there is an infinite number of matrices $X$ with rank $r$ with the given entries [22], so the $F R$ varies in $(0,1)$ in this paper.

In this subsection, we applied the proposed nonconvex method (ADMMdp) for solving the matrix completion problem (1.6). In order to illustrate the performance of this method, we compare the proposed nonconvex method (ADMMdp) with the MCP regularized method [22], SCAD regularized method, Capped- $\ell_{1}$ regularized method, the state-ofthe-art method APGL [39] (The code is downloaded from http://www.math.nus.edu.sg/ mattohkc/NNLS.html.) and PD [27] (The code is downloaded from http://www.sfu.ca/ yza30 /homepage/PD_ Rank/downloads.html.). MCP, SCAD and Capped- $\ell_{1}$ are listed in Table 1 and the corresponding problems read

$$
\min _{X \in \mathbb{R}^{n_{1} \times n_{2}}}\left\{\frac{1}{2}\|\mathcal{A} W-\mathbf{b}\|^{2}+\sum_{i=1}^{m} \rho_{\lambda, \tau}\left(\sigma_{i}(X)\right): X=W\right\} .
$$

We use the ADMM to solve them which are used to solve the MCP regularized CS problem in the above subsection. In all tests, let $X^{\text {opt }}$ be the optimal solution produced the the proposed nonconvex method, we use the relative estimation error to measure the quality of $X^{\text {opt }}$ to original $X$, in particular,

$$
\text { RelErr }=\frac{\left\|X^{o p t}-X\right\|_{F}}{\|X\|_{F}} .
$$


And we use the RelErr $=1 e-4$ as the terminal condition.

Firstly, we test them for the easy matrix completion problems and report the numerical results in Table 3. And from Table 3 it is clear to see that the ADMMdp can get higher accuracy than the others. For further illustrate the efficiency of The ADMMdp, we test them in the following hard matrix completion problems. The results are shown in the Table 4, we can see that the ADMMdp performs better than APGL, but when the dimension becomes higher, the APGL is faster than ADMMdp, MPC (ADMc) and Capped $\ell_{1}$. By these limited tests, we can see that the proposed ADMMdp is comprarable with MPC (ADMc), Capped- $\ell_{1}$ and APGL for solving hard completion problems.

In some practical applications, the observations may contain Gaussian noise. For this situation, we test them for some easy and hard matrix completion problems with noise level $\sigma=1 e-3$ in the following tests. From Table 5, we know that our proposed ADMMdp also performs well for the low rank matrix completion problems with noise.

Table 3: Numerical result of ADMMdp, MCP (ADM), SCAD and PD for easy matrix completion with

\begin{tabular}{|c|c|c|c|c|c|c|c|c|c|}
\hline \multirow[t]{2}{*}{$\left(n_{1}, r\right)$} & \multirow[t]{2}{*}{$n_{3} / d r$} & \multicolumn{2}{|c|}{ ADMMdp } & \multicolumn{2}{|c|}{$\mathrm{MCP}(\mathrm{ADM})$} & \multicolumn{2}{|c|}{$\mathrm{SCAD}$} & \multicolumn{2}{|c|}{$\mathrm{PD}$} \\
\hline & & Time & RelErr & Time & RelErr & Time & RelErr & Time & RelErr \\
\hline$(200,10)$ & 5.128 & 3.37 & $5.60 \mathrm{e}-06$ & 2.86 & $8.79 \mathrm{e}-06$ & 3.12 & $9.26 \mathrm{e}-06$ & 6.38 & $9.51 \mathrm{e}-06$ \\
\hline$(300,10)$ & 7.627 & 4.18 & $5.25 \mathrm{e}-06$ & 3.48 & $8.92 \mathrm{e}-06$ & 4.28 & $9.58 \mathrm{e}-06$ & 12.52 & $7.94 \mathrm{e}-06$ \\
\hline$(400,10)$ & 10.127 & 7.46 & $5.15 \mathrm{e}-06$ & 6.17 & $7.58 \mathrm{e}-06$ & 6.35 & $7.21 \mathrm{e}-06$ & 28.21 & $8.83 \mathrm{e}-06$ \\
\hline$(500,10)$ & 12.626 & 11.04 & $5.07 \mathrm{e}-06$ & 8.96 & $7.67 \mathrm{e}-06$ & 9.91 & $7.57 \mathrm{e}-06$ & 54.91 & $8.31 \mathrm{e}-06$ \\
\hline$(600,10)$ & 15.126 & 15.39 & $4.84 \mathrm{e}-06$ & 12.3 & $8.25 \mathrm{e}-06$ & 13.1 & $8.65 \mathrm{e}-06$ & 95.32 & $7.59 \mathrm{e}-06$ \\
\hline$(700,10)$ & 17.626 & 21.19 & $4.79 \mathrm{e}-06$ & 14.05 & $5.65 \mathrm{e}-06$ & 15.28 & $5.66 \mathrm{e}-06$ & 175.28 & $7.30 \mathrm{e}-06$ \\
\hline$(800,10)$ & 20.126 & 29.57 & $4.85 \mathrm{e}-07$ & 18.22 & $7.03 \mathrm{e}-06$ & 20.47 & $7.34 \mathrm{e}-06$ & 267.39 & $6.32 \mathrm{e}-06$ \\
\hline$(900,10)$ & 22.626 & 35.48 & $4.76 \mathrm{e}-07$ & 20.35 & $5.87 \mathrm{e}-06$ & 24.17 & $6.17 \mathrm{e}-06$ & 283.34 & $8.90 \mathrm{e}-06$ \\
\hline$(1000,10)$ & 25.126 & 39.27 & $3.89 \mathrm{e}-07$ & 25.81 & $9.45 \mathrm{e}-06$ & 28.27 & $8.86 \mathrm{e}-06$ & 504.68 & $8.99 \mathrm{e}-06$ \\
\hline
\end{tabular}


Table 4: Numerical result of ADMMdp, MCP (ADMc), Capped- $\ell_{1}$ and APGL for easy matrix completion

\begin{tabular}{|c|c|c|c|c|c|c|c|c|c|}
\hline \multirow[t]{2}{*}{$\left(n_{1}, r\right)$} & \multirow[t]{2}{*}{$n_{3} / d r$} & \multicolumn{2}{|c|}{ ADMMdp } & \multicolumn{2}{|c|}{ MCP (ADMc) } & \multicolumn{2}{|c|}{ Capped- $\ell_{1}$} & \multicolumn{2}{|c|}{ APGL } \\
\hline & & Time & RelErr & Time & RelErr & Time & RelErr & Time & RelErr \\
\hline$(100,10)$ & 2.632 & 2.73 & $6.83 \mathrm{e}-06$ & 2.41 & $2.41 \mathrm{e}-06$ & 2.45 & $6.45 \mathrm{e}-06$ & 1.38 & $5.22 \mathrm{e}-04$ \\
\hline$(100,15)$ & 1.802 & 3.50 & $6.57 \mathrm{e}-06$ & 3.32 & $4.12 \mathrm{e}-06$ & 3.64 & $6.72 \mathrm{e}-06$ & 1.52 & $4.27 \mathrm{e}-04$ \\
\hline$(200,20)$ & 2.632 & 6.97 & $1.11 \mathrm{e}-06$ & 6.52 & $1.39 \mathrm{e}-06$ & 6.27 & $6.47 \mathrm{e}-06$ & 2.21 & $3.86 \mathrm{e}-04$ \\
\hline$(200,25)$ & 2.133 & 8.01 & $6.61 \mathrm{e}-06$ & 7.15 & $8.94 \mathrm{e}-06$ & 6.37 & $6.92 \mathrm{e}-06$ & 2.91 & $1.30 \mathrm{e}-04$ \\
\hline$(300,30)$ & 2.632 & 14.01 & $1.28 \mathrm{e}-06$ & 12.50 & $1.47 \mathrm{e}-06$ & 12.12 & $3.25 \mathrm{e}-06$ & 3.32 & $6.40 \mathrm{e}-05$ \\
\hline$(300,35)$ & 2.276 & 16.59 & $1.50 \mathrm{e}-06$ & 14.81 & $2.16 \mathrm{e}-06$ & 14.53 & $2.63 \mathrm{e}-06$ & 3.28 & $3.99 \mathrm{e}-04$ \\
\hline$(400,40)$ & 2.632 & 24.64 & $1.38 \mathrm{e}-06$ & 24.70 & $1.54 \mathrm{e}-06$ & 23.25 & $1.89 \mathrm{e}-06$ & 5.39 & $2.62 \mathrm{e}-04$ \\
\hline$(400,45)$ & 2.355 & 25.52 & $1.72 \mathrm{e}-06$ & 37.33 & $1.59 \mathrm{e}-06$ & 29.31 & $2.63 \mathrm{e}-06$ & 6.34 & $6.75 \mathrm{e}-05$ \\
\hline$(500,50)$ & 2.632 & 39.27 & $1.42 \mathrm{e}-06$ & 38.39 & $1.62 \mathrm{e}-06$ & 37.22 & $1.81 \mathrm{e}-06$ & 7.28 & $1.98 \mathrm{e}-04$ \\
\hline$(500,55)$ & 2.405 & 39.60 & $1.44 \mathrm{e}-06$ & 54.56 & $1.45 \mathrm{e}-06$ & 41.09 & $1.52 \mathrm{e}-06$ & 9.63 & $9.09 \mathrm{e}-05$ \\
\hline$(600,60)$ & 2.632 & 58.65 & $1.53 \mathrm{e}-06$ & 56.34 & $1.52 \mathrm{e}-06$ & 56.72 & $1.79 \mathrm{e}-06$ & 10.48 & $3.18 \mathrm{e}-04$ \\
\hline$(700,70)$ & 2.632 & 85.08 & $1.41 \mathrm{e}-06$ & 79.78 & $1.50 \mathrm{e}-06$ & 74.71 & $1.52 \mathrm{e}-06$ & 17.10 & $3.04 \mathrm{e}-04$ \\
\hline$(800,80)$ & 2.632 & 114.73 & $1.34 \mathrm{e}-06$ & 108.18 & $1.15 \mathrm{e}-06$ & 105.59 & $1.45 \mathrm{e}-06$ & 26.26 & $4.36 \mathrm{e}-05$ \\
\hline$(900,90)$ & 2.632 & 151.09 & $1.52 \mathrm{e}-06$ & 145.86 & $1.44 \mathrm{e}-06$ & 128.17 & $1.54 \mathrm{e}-06$ & 38.51 & $9.70 \mathrm{e}-05$ \\
\hline$(1000,100)$ & 2.632 & 219.27 & $1.43 \mathrm{e}-06$ & 182.23 & $1.51 \mathrm{e}-06$ & 179.65 & $1.53 \mathrm{e}-06$ & 49.68 & $9.94 \mathrm{e}-06$ \\
\hline
\end{tabular}

Finally, we test the proposed nonconvex method in recovering two real corrupted gray scale images. At first, we use SVD to obtain the low-rank-50 images. Then we randomly select $40 \%$ samples from the low-rank images, which are corrupted with noise level $\sigma=1 e-3$. Finally, these corrupted images are recovered by the proposed ADMMdp, MCP, SCAD, Capped- $\ell_{1}$ and the SVT model [3]. From Fig. 2, it shows that the quality of image restored by proposed nonconvex method ADMMdp are better than the others.

From the above tests, it can be seen that matrix completion algorithms with the nonconvex penalty functions achieve generally much better recovery performance than the convex methods, i.e, PD, APGL and SVT. This is because that the nonconvex penalty functions approximate the rank function better than the convex nuclear norm. What's more, from Table 2-4 and Fig. 2, we known that our ADMMdp performs generally better than the nonconvex methods, i.e., MCP, SCAD and Capped- $\ell_{1}$, since the solutions which obtained by the three methods are not globally optimal. In our algorithm (see Algorithm 
Table 5: Numerical result of ADMMdp, MCP (ADMc), SCAD and APGL for easy matrix completion

\begin{tabular}{ccccccccccc}
\multicolumn{1}{c}{ with noise $\sigma=1 e-3}$. \\
\hline \hline$\left(n_{1}, r\right)$ & $s r$ & $n_{3} / d r$ & \multicolumn{2}{c}{ ADMMdp } & \multicolumn{2}{c}{ MCP } & (ADMc) & \multicolumn{2}{c}{ SCAD } & \multicolumn{2}{c}{ APGL } \\
& & & Time & RelErr & Time & RelErr & Time & RelErr & Time & RelErr \\
\hline$(300,30)$ & 0.5 & 2.632 & 3.27 & $1.63 \mathrm{e}-04$ & 2.41 & $1.42 \mathrm{e}-04$ & 3.16 & $1.85 \mathrm{e}-04$ & 1.38 & $5.22 \mathrm{e}-04$ \\
& 0.7 & 3.684 & 3.51 & $1.02 \mathrm{e}-04$ & 3.32 & $1.00 \mathrm{e}-04$ & 3.63 & $1.21 \mathrm{e}-04$ & 1.52 & $4.27 \mathrm{e}-04$ \\
$(500,50)$ & 0.5 & 2.632 & 9.06 & $8.96 \mathrm{e}-05$ & 7.15 & $9.99 \mathrm{e}-05$ & 8.63 & $1.03 \mathrm{e}-04$ & 2.91 & $1.30 \mathrm{e}-04$ \\
& 0.7 & 3.684 & 7.63 & $8.90 \mathrm{e}-05$ & 12.50 & $9.16 \mathrm{e}-05$ & 13.47 & $9.52 \mathrm{e}-05$ & 3.32 & $6.40 \mathrm{e}-05$ \\
$(700,70)$ & 0.5 & 2.632 & 20.04 & $9.63 \mathrm{e}-05$ & 24.70 & $9.71 \mathrm{e}-05$ & 26.21 & $9.51 \mathrm{e}-05$ & 5.39 & $2.62 \mathrm{e}-04$ \\
& 0.7 & 3.684 & 15.37 & $8.14 \mathrm{e}-05$ & 37.33 & $7.88 \mathrm{e}-05$ & 23.51 & $8.79 \mathrm{e}-05$ & 6.34 & $6.75 \mathrm{e}-05$ \\
$(900,90)$ & 0.5 & 2.632 & 45.71 & $9.41 \mathrm{e}-05$ & 54.56 & $9.48 \mathrm{e}-05$ & 52.26 & $9.53 \mathrm{e}-05$ & 9.63 & $9.09 \mathrm{e}-05$ \\
& 0.7 & 3.684 & 27.64 & $7.54 \mathrm{e}-05$ & 56.34 & $7.46 \mathrm{e}-05$ & 53.51 & $8.21 \mathrm{e}-05$ & 10.48 & $3.18 \mathrm{e}-04$ \\
\hline \hline
\end{tabular}

1), at every iterations, we make only one gradient descent step with Quasi-Newtons method for $X$. In other words, our algorithm can avoid to fall into the local minimizers of the cost function too early, which the three nonconvex methods, i.e., MCP, SCAD and Capped- $\ell_{1}$ can't avoid it. Another thing worth noting is that all the subproblems in our algorithm are convex or smooth, which we think have contributed to the high performance of our algorithm, its strict theoretical proof remains to be studied deeply.
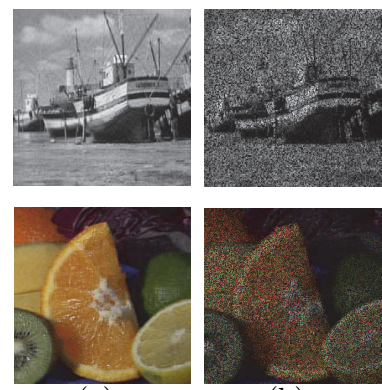

(a)

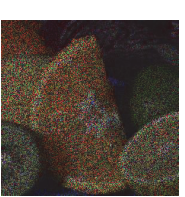

(b)
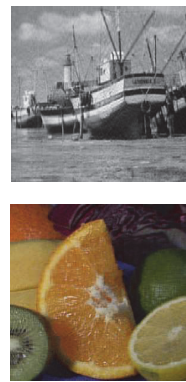

(c)
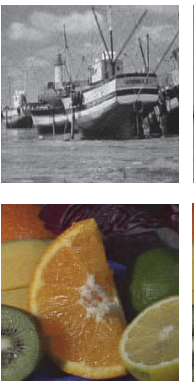

(d)
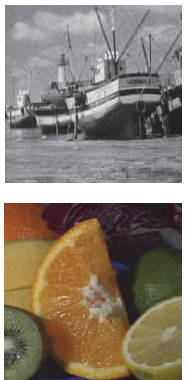

(e)
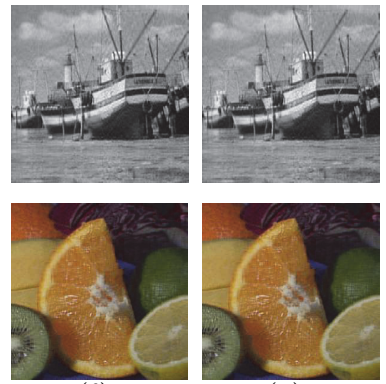

(f)

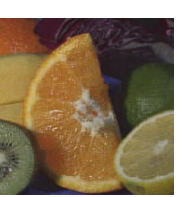

(g)

Figure 2: (a) Corresponding low rank image with $n_{1}=n_{2}=512, r=40$ and downsamplefactor=1.8; (b) Randomly masked images from rank 50 with $s r=50 \%, \sigma=1 e-3$; (c) Recovered images by ADMMdp [ErrRel=3.24e-03 (first image), 1.54e-02 (second image)]; (d) Recovered images by MCP [ErrRel=4.51e03 (first image), 1.69e-02 (second image)]; (e) Recovered images by SCAD [ErrRel=3.86e-03 (first image), 1.91e-02 (second image)]; (f) Recovered images by Capped- $\ell_{1}$ [ErrRel=6.56e-03 (first image), $2.26 \mathrm{e}-02$ (second image)]; (g) Recovered images by SVT [ErrRel=1.81e-02 (first image), 4.05e-02 (second image)]. 


\section{Conclusion}

In this paper, we gave a nonconvex and nonsmooth method for CS and MC and developed an alternating minimization scheme for solving it. Then, we proved the convergence of the sequence which generate by the alternating minimization algorithm. In addition, we showed the recovery guarantees for our proposed method, including NSP and RIP. At last, we given some numerical experiments that perform well for our proposed method with simulated and real data. However, our model didn't consider the correlations of all the singular values. In the future research, we desire to measure the low-rank property of $\mathrm{X}$ at group level in which the all singular values are nonzero (or zero) simultaneously, and to give theoretical analysis for the recovery guarantees at group level.

\section{Acknowledgment}

We thank the anonymous referees for their detailed comments, which helped to improve the paper.

\section{Appendix}

(A) Proof of Proposition 2.1. $\varphi$ is a nonconvex and nonsmooth function, and $\varphi(t)=$ $\alpha|t|+\psi(t), \psi \in \mathcal{C}^{2}$. By the chain rule of Gateaux derivative, we have

$$
D_{X} \hat{\Psi}(X)=\left\langle D_{\Sigma} \hat{\Psi}(X), D_{X} \Sigma\right\rangle,
$$

where $\Sigma=\operatorname{diag}\left(\sigma_{1}, \ldots, \sigma_{m}\right)_{n_{1} \times n_{2}} \in \mathbb{R}^{n_{1} \times n_{2}}$. Via the definition of Gateaux derivative, we obtain

$$
D_{\Sigma} \hat{\Psi}(\Sigma)=\lim _{t \rightarrow 0} \frac{1}{t}\left|\hat{\Psi}\left(\Sigma+t \Sigma^{\prime}\right)-\hat{\Psi}(\Sigma)-t\left\langle D_{\Sigma} \hat{\Psi}(\Sigma), \Sigma^{\prime}\right\rangle \mathcal{X}\right|=0
$$

where $\Sigma^{\prime} \in \mathbb{R}^{n_{1} \times n_{2}}$ is an arbitrarily chosen diagonal matrix. We have

$$
D_{\Sigma} \hat{\Psi}(\Sigma)=\operatorname{diag}\left(\psi^{\prime}\left(\sigma_{1}\right), \ldots, \psi^{\prime}\left(\sigma_{m}\right)\right)_{n_{1} \times n_{2}}
$$

For $D_{X} \Sigma$ in (5.1), via the definition of Gateaux derivative, we have

$$
\left\langle D_{X} \Sigma(X), H\right\rangle=U H V^{*},
$$


where $U$ and $V$ are unitary matrices which consist of the left-singular vectors and rightsingular vectors, respectively, and $\Sigma(X) \in \mathbb{R}^{n_{1} \times n_{2}} \rightarrow \mathbb{R}^{n_{1} \times n_{2}}, D_{X} \Sigma(X) \in \mathbb{R}^{n_{1} \times n_{2}} \rightarrow$ $L\left(\mathbb{R}^{n_{1} \times n_{2}}\right)$ and $H \in \mathbb{R}^{n_{1} \times n_{2}}$ is an arbitrary matrix. Applying (5.3) and (5.2) to (5.1), we have

$$
D_{X} \hat{\Psi}(X)=U \operatorname{diag}\left(\psi^{\prime}\left(\sigma_{1}\right), \ldots, \psi^{\prime}\left(\sigma_{m}\right)\right)_{n_{1} \times n_{2}} V^{*} .
$$

(в) Proof of Proposition 2.2. The inequalities (2.6) and (2.8) can be obtained by direct computation. The proof for the inequality of (2.7) can be considered as follows.

$$
\begin{aligned}
& L_{\rho}\left(X^{(s+1)}, W^{(s+1)}, Z^{(s)}\right) \leq L_{\rho}\left(X^{(s)}, W^{(s+1)}, Z^{(s)}\right) \\
& \quad+\left\langle D_{X} L_{\rho}\left(X^{(s)}, W^{(s+1)}, Z^{(s)}\right), X^{(s+1)}-X^{(s)}\right\rangle_{\mathcal{X}}+\frac{L}{2}\left\|X^{(s+1)}-X^{(s)}\right\|_{F}^{2},
\end{aligned}
$$

where $L$ is a Lipschitz constant of $D_{X} L_{\rho}\left(X^{(s)}, W^{(s+1)}, Z^{(s)}\right)$. By (2.5), we have

$$
\begin{aligned}
& L_{\rho}\left(X^{(s+1)}, W^{(s+1)}, Z^{(s)}\right) \leq L_{\rho}\left(X^{(s)}, W^{(s+1)}, Z^{(s)}\right) \\
& \quad-\left\langle\left(\mathcal{A}^{*} \mathcal{A}+\rho \mathcal{I}\right)\left(X^{(s+1)}-X^{(s)}\right), X^{(s+1)}-X^{(s)}\right\rangle \mathcal{X}+\frac{L}{2}\left\|X^{(s+1)}-X^{(s)}\right\|_{F}^{2} .
\end{aligned}
$$

We have

$$
L_{\rho}\left(X^{(s+1)}, W^{(s+1)}, Z^{(s)}\right)+\left(\underline{\lambda}+\rho-\frac{L}{2}\right)\left\|X^{(s+1)}-X^{(s)}\right\|_{F}^{2} \leq L_{\rho}\left(X^{(s)}, W^{(s+1)}, Z^{(s)}\right) .
$$

By the assumption of the proposition, i.e, $\underline{\lambda}+\rho-\frac{L}{2}>0$, then the inequality (2.7) holds.

(c) Proof of Theorem 2.1. According to (2.3) and (2.5), we have

$$
\begin{aligned}
\mathbf{0} & \in \partial_{W} L_{\rho}\left(X^{(s)}, W^{(s+1)}, Z^{(s)}\right) \\
& =\lambda \alpha \partial\left\|W^{(s+1)}\right\|_{*}+Z^{(s)}-\rho\left(W^{(s+1)}-X^{(s)}\right),
\end{aligned}
$$

and

$$
\begin{aligned}
- & \left(\mathcal{A}^{*} \mathcal{A}+\rho \mathcal{I}\right)\left(X^{(s+1)}-X^{(s)}\right)=D L_{\rho}\left(X^{(s)}, W^{(s+1)}, Z^{(s)}\right) \\
& =\mathcal{A}^{*}\left(\mathcal{A} X^{(s)}-\mathbf{b}\right)+\lambda D \hat{\Psi}\left(X^{(s)}\right)-Z^{(s)}+\rho\left(W^{(s+1)}-X^{(s)}\right) .
\end{aligned}
$$

By the definition of the augmented Lagrangian function (2.2), we have

$$
\begin{gathered}
\partial_{W} L_{\rho}\left(X^{(s+1)}, W^{(s+1)}, Z^{(s+1)}\right) \\
=\lambda \alpha \partial\left\|W^{(s+1)}\right\|_{*}+Z^{(s+1)}-\rho\left(W^{(s+1)}-X^{(s+1)}\right), \\
D_{X} L_{\rho}\left(X^{(s+1)}, W^{(s+1)}, Z^{(s+1)}\right) \\
=\mathcal{A}^{*}\left(\mathcal{A} X^{(s+1)}-\mathbf{b}\right)+\lambda D \hat{\Psi}\left(X^{(s+1)}\right)-Z^{(s+1)}+\rho\left(W^{(s+1)}-X^{(s+1)}\right),
\end{gathered}
$$


With (5.4) and (5.6), we obtain

$$
\partial_{W} L_{\rho}\left(X^{(s+1)}, W^{(s+1)}, Z^{(s+1)}\right)=Z^{(s+1)}-Z^{(s)}+\rho\left(X^{(s+1)}-X^{(s)}\right) .
$$

With (5.5) and (5.7), we obtain

$$
D_{X} L_{\rho}\left(X^{(s+1)}, W^{(s+1)}, Z^{(s+1)}\right)=\lambda D \hat{\Psi}\left(X^{(s+1)}\right)-\lambda D \hat{\Psi}\left(X^{(s)}\right)+Z^{(s)}-Z^{(s+1)} .
$$

And via (2.2), we have

$$
D_{Z} L_{\rho}\left(X^{(s+1)}, W^{(s+1)}, Z^{(s+1)}\right)=\frac{1}{\rho}\left(Z^{(s+1)}-Z^{(s)}\right) .
$$

From above three equalities, we known that there exist a $\kappa>0$, and $\kappa \cdot \operatorname{dist}\left(\partial L_{\rho}\left(X^{(s+1)}, W^{(s+1)}, Z^{(s+1)}\right), 0\right)^{2} \leq L_{\rho}\left(X^{(s)}, W^{(s)}, Z^{(s)}\right)-L_{\rho}\left(X^{(s+1)}, W^{(s+1)}, Z^{(s+1)}\right)$.

300 Then, (2.9) holds.

(D) Proof of Theorem 2.2. From (2.6), (2.7) and (2.8), we know that the sufficient decrease condition [1] is satisfied. Therefore we only need to prove

(1) relative error condition, i.e., there exists

$$
\left(\widehat{X}^{(s+1)}, \widehat{W}^{(s+1)}, \widehat{Z}^{(s+1)}\right) \in \partial L_{\rho}\left(X^{(s+1)}, W^{(s+1)}, Z^{(s+1)}\right)
$$

such that

$$
\begin{aligned}
\left\|\widehat{X}^{(s+1)}\right\|_{F} & +\left\|\widehat{W}^{(s+1)}\right\|_{F}+\left\|\widehat{Z}^{(s+1)}\right\|_{F} \\
& \leq b\left(\left\|X^{(s+1)}-X^{(s)}\right\|_{F}+\left\|W^{(s+1)}-W^{(s)}\right\|_{F}+\left\|Z^{(s+1)}-Z^{(s)}\right\|_{F}\right) .
\end{aligned}
$$

(2) continuity condition, i.e., there exists a subsequence $\left(u^{\left(s_{j}\right)}, W^{\left(s_{j}\right)}, P^{\left(s_{j}\right)}\right)$ such that

$$
\begin{aligned}
& \left(X^{(s)}, W^{(s)}, Z^{(s)}\right) \rightarrow\left(X^{*}, W^{*}, Z^{*}\right), \\
& L_{\rho}\left(X^{(s)}, W^{(s)}, Z^{(s)}\right) \rightarrow L_{\rho}\left(X^{*}, W^{*}, Z^{*}\right) \text {, as } j \rightarrow+\infty .
\end{aligned}
$$

First, define

$$
\left\{\begin{array}{l}
\widehat{X}^{(s+1)}=Z^{(s+1)}-Z^{(s)}+\rho\left(X^{(s+1)}-X^{(s)}\right) \\
\widehat{W}^{(s+1)}=\lambda D \hat{\Psi}\left(X^{(s+1)}\right)-\lambda D \hat{\Psi}\left(X^{(s)}\right)+Z^{(s)}-Z^{(s+1)} \\
\widehat{Z}^{(s+1)}=\frac{1}{\rho}\left(Z^{(s+1)}-Z^{(s)}\right) .
\end{array}\right.
$$


Let $b=\lambda L^{\prime}+\rho+\frac{1}{\rho}+2$, then, (5.8) holds, where $D \hat{\Psi}$ is $L^{\prime}$-Lipschitz function. By the continuity of $L_{\rho}(X, W, Z)$, it is easy to know that (5.9) also holds.

(e) Proof of equation 4.5. For MCP, from the Definition 1.3 we can compute $\bar{\partial} \rho_{\lambda, \tau}$, where $\rho_{\lambda, \tau}$ defined in (4.2). Since for all $x_{i} \neq 0, \rho_{\lambda, \tau}$ is differentiable, we have

$$
\forall x_{i} \neq 0, \bar{\partial} \rho_{\lambda, \tau}\left(x_{i}\right)= \begin{cases}\lambda\left(\operatorname{sign}\left(x_{i}\right)-\frac{x_{i}}{\lambda \tau}\right) & \text { if }\left|x_{i}\right|<\tau \lambda, \\ 0 & \text { if }\left|x_{i}\right| \geq \tau \lambda .\end{cases}
$$

The remaining case is $x_{i}=0$. From the definition of generalized direction derivative at $x_{i}=0$, we have

$$
\begin{aligned}
\forall v \in \mathbb{R}, \bar{d}(0, v) & =\limsup _{y \rightarrow 0} \frac{\rho_{\lambda, \tau}(y+\eta v)-\rho_{\lambda, \tau}(y)}{\eta} \\
& =\limsup _{y \rightarrow 0} \frac{\lambda}{\eta \downarrow 0}\left(|y+\eta v|-\frac{(y+\eta v)^{2}}{2 \lambda \tau}-|y|+\frac{y^{2}}{2 \lambda \tau}\right) .
\end{aligned}
$$

Then we have

$$
\bar{d}(0, v)=\lambda|v| .
$$

Therefore, from the definition of generalized gradient, $\bar{\partial} \rho_{\lambda, \tau}(0)$ contains all $\xi$ verifying $\lambda|v| \geq \xi v$, that is,

$$
\bar{\partial} \rho_{\lambda, \tau}(0)=[-\lambda, \lambda] .
$$

On the other hand, if $\mathbf{x}$ is a minimum of (4.4), we have

$$
\mathbf{0} \in \rho\left(\mathbf{x}-\widehat{\mathbf{x}}^{(s)}\right)+\bar{\partial} \sum_{i=1}^{p} \rho_{\lambda, \tau}\left(x_{i}\right)
$$

thus, via (5.10), we have

$$
\begin{aligned}
& \left\{\begin{array}{lr}
0 \in\left[x_{i}-\widehat{x}_{i}^{(s)}-\lambda / \rho, x_{i}-\widehat{x}_{i}^{(s)}+\lambda / \rho\right], & \text { iff } x_{i}=0 \\
x_{i}-\widehat{x}_{i}^{(s)}+\frac{\lambda}{\rho}\left[\operatorname{sign}\left(x_{i}\right)-\frac{x_{i}}{\lambda \tau}\right]=0, & \text { iff } 0<\left|x_{i}\right| \leq \tau \lambda \\
x_{i}-\widehat{x}_{i}^{(s)}=0 . & \text { iff }\left|x_{i}\right|>\tau \lambda
\end{array}\right. \\
& \Longleftrightarrow\left\{\begin{array}{lr}
x_{i}=0, & \text { iff }\left|\widehat{x}_{i}^{(s)}\right| \leq \lambda / \rho \\
x_{i}=\operatorname{sign}\left(\widehat{x}_{i}^{(s)}\right) \frac{\tau\left(\rho\left|\widehat{x}_{i}^{(s)}\right|-\lambda\right)}{\rho \tau-1}, & \text { iff } \lambda / \rho<\left|\widehat{x}_{i}^{(s)}\right| \leq \tau \lambda \\
x_{i}=\widehat{x}_{i}^{(s)} . & \text { iff }\left|\widehat{x}_{i}^{(s)}\right|>\tau \lambda
\end{array}\right.
\end{aligned}
$$

It is easy to get the solutions for SCAD and Capped- $\ell_{1}$ to use the same method. 


\section{References}

[1] H. Attouch, J. Bolte, P. Redont, A. Soubeyran, Proximal alternating minimization and projection methods for nonconvex problems: an approach based on the Kurdyka-Łojasiewicz inequality, Math. Oper. Res. 35 (2010) 438-457.

[2] J. Cai, S. Osher, Z. Shen, Linearized bregman iterations for compressed sensing, Mathematics of Computation 78 (2009) 1515-1536.

[3] J.-F. Cai, E. J. Candès, Z. Shen, A singular value thresholding algorithm for matrix completion, SIAM J. Optim. 20 (2010) 1956-1982.

[4] E. J. Candès, B. Recht, Exact matrix completion via convex optimization, Found. Comput. math. 9 (2009) 717-772.

[5] E. J. Candès, J. Romberg, T. Tao, Robust uncertainty principles: Exact signal reconstruction from highly incomplete frequency information, IEEE Trans. Inform. Theory 52 (2006) 489-509.

[6] E. J. Candès, J. K. Romberg, T. Tao, Stable signal recovery from incomplete and inaccurate measurements, Commun. pure appl. math. 59 (2006) 1207-1223.

[7] E. J. Candès, T. Tao, Decoding by linear programming, IEEE Trans. Inform. Theory 51 (2005) $4203-4215$.

[8] E. J. Candès, T. Tao, Near-optimal signal recovery from random projections: Universal encoding strategies?, IEEE Trans. Inform. Theory 52 (2006) 5406-5425.

[9] E. J. Candès, T. Tao, The power of convex relaxation: Near-optimal matrix completion, IEEE Trans. Inform. Theory 56 (2010) 2053-2080.

[10] E. J. Candès, M. B. Wakin, An introduction to compressive sampling, IEEE Signal Processing Magazine 25 (2008) 21-30.

[11] X. Chen, W. Zhou, Smoothing nonlinear conjugate gradient method for image restoration using nonsmooth nonconvex minimization, SIAM J. Imag. Sci. 3 (2010) 765-790.

[12] D. L. Donoho, X. Huo, Uncertainty principles and ideal atomic decompositions, IEEE Trans. Inform. Theory 47 (2001) 2845-2862.

[13] J. Fan, R. Li, Variable selection via nonconcave penalized likelihood and its oracle properties, J. Am. Stat. Assoc. 96 (2001) 1348-1360.

[14] S. Foucart, H. Rauhut, A Mathematical Introduction to Compressive Sensing, Basel: Birkha̋user, 2013.

[15] L. E. Frank, J. H. Friedman, A statistical view of some chemometrics regression tools, Technometrics 35 (1993) 109-135.

[16] J. H. Friedman, Fast sparse regression and classification, International Journal of Forecasting 28 (2012) $722-738$.

[17] C. Gao, N. Wang, Q. Yu, Z. Zhang, A feasible nonconvex relaxation approach to feature selection, in: AAAI, 2011.

[18] D. Geman, C. Yang, Nonlinear image recovery with half-quadratic regularization, IEEE Trans. Image Proces. 4 (1995) 932-946. 
[19] S. Ghadimi, G. Lan, Stochastic first- and zeroth-order methods for nonconvex stochastic programming, SIAM J. Optim. 23 (2013) 2341-2368.

[20] R. Gribonval, M. Nielsen, Sparse representations in unions of bases, IEEE Trans. Inform. Theory 49 (2003) 3320-3325.

[21] Y. Hu, D. Zhang, J. Ye, X. Li, X. He, Fast and accurate matrix completion via truncated nuclear norm regularization, IEEE Trans. Pattern Anal. Mach. Intell. 35 (2013) 2117-2130.

[22] Z.-F. Jin, Z. Wan, Y. Jiao, X. Lu, An alternating direction method with continuation for nonconvex low rank minimization, J. Sci. Comput. 66 (2016) 849-869.

[23] M.-J. Lai, Y. Xu, W. Yin, Improved iteratively reweighted least squares for unconstrained smoothed $\ell_{q}$ minimization, SIAM J. Numer. Anal. 51 (2013) 927-925.

[24] M.-J. Lai, W. Yin, Augmented $\ell_{1}$ and nuclear-norm models with a globally linearly convergent algorithm, SIAM J. Imag. Sci. 6 (2013) 1059-1091.

[25] Y.-F. Li, Y.-J. Zhang, Z.-H. Huang, A reweighted nuclear norm minimization algorithm for low rank matrix recovery, J. Comput. Appl. Math. 263 (2014) 338-350.

[26] C. Lu, J. Tang, S. Yan, Z. Lin, Nonconvex nonsmooth low rank minimization via iteratively reweighted nuclear norm, IEEE Trans. Image Process. 25 (2016) 829-839.

[27] Z. Lu, Y. Zhang, X. Li, Penalty decomposition methods for rank minimization, Optimization Methods and Software 30 (2015) 531-558.

[28] M. Malek-Mohammadi, M. Babaie-Zadeh, A. Amini, C. Jutten, Recovery of low-rank matrices under affine constraints via a smoothed rank function, IEEE Trans. Signal Process. 62 (2014) 981-992.

[29] M. Malek-Mohammadi, C. R. Rojas, B. Wahlberg, A class of nonconvex penalties preserving overall convexity in optimization-based mean filtering, IEEE Trans. Signal Proces. 64 (2016) 6650-6664.

[30] Q. Mo, S. Li, New bounds on the restricted isometry constant $\delta_{2 k}$, Appl. Comput. Harmon. Anal. 31 (2011) 460-468.

[31] B. K. Natarajan, Sparse approximate solutions to linear systems, SIAM J. Comput. 24 (1995) $227-234$.

[32] M. Nikolova, M. K. Ng, C.-P. Tam, Fast nonconvex nonsmooth minimization methods for image restoration and reconstruction, IEEE Trans. Image Process. 19 (2010) 3073-3088.

[33] M. Nikolova, M. K. Ng, C.-P. Tam, On $\ell_{1}$ data fitting and concave regularization for image recovery, SIAM J. Sci. Comput. 35 (2013) A397-A430.

[34] J. M. Ortega, W. C. Rheinboldt, Iterative Solution of Nonlinear Equations in Several Variables, vol. 30, SIAM, 1970.

[35] A. Parekh, I. W. Selesnick, Enhanced low-rank matrix approximation, IEEE Signal Processing Letters 23 (2015) 493-497.

[36] B. Recht, M. Fazel, P. A. Parrilo, Guaranteed minimum-rank solutions of linear matrix equations via nuclear norm minimization, SIAM review 52 (2010) 471-501.

[37] I. W. Selesnick, A. Parekh, I. Bayram, Convex 1-d total variation denoising with non-convex regularization, IEEE Signal Processing Letters 22 (2015) 141-144.

[38] R. Tibshirani, Regression shrinkage and selection via the lasso, Journal of the Royal Statistical 
Society 58 (1996) 267-288.

[39] K.-C. Toh, S. Yun, An accelerated proximal gradient algorithm for nuclear norm regularized linear least squares problems, Pac. J. Optim. 15 (2010) 615-640.

[40] J. D. Trzasko, A. Manduca, Highly undersampled magnetic resonance image reconstruction via homotopic $\ell_{0}$-minimization, IEEE Trans. Medical Imag. 1 (2009) 106-121.

[41] G. A. Watson, Characterization of the subdifferential of some matrix norms, Linear algebra and its applications 170 (1992) 33-45.

[42] J. Xiao, M. K.-P. Ng, Y.-F. Yang, On the convergence of nonconvex minimization methods for image recovery, IEEE Trans. Image Process. 24 (2015) 1587-1598.

[43] C. Zhang, Nearly unbiased variable selection under minimax concave penalty, Annals of Statistics 38 (2010) 894-942.

[44] C.-H. Zhang, Nearly unbiased variable selection under minimax concave penalty, The Annals of Statistics (2010) 894-942.

[45] T. Zhang, Analysis of multi-stage convex relaxation for sparse regularization, J. Mach. Learn. Res. 11 (2010) 1081-1107.

[46] M. V. W. Zibetti, D. R. Pipa, A. R. De Pierro, Fast and exact unidimensional L2-L1 optimization as an accelerator for iterative reconstruction algorithms, Digital Signal Process. 48 (2016) 178-187.

\section{Vitae}

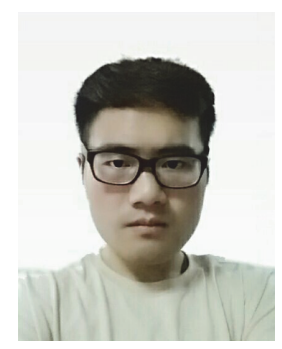

Zhuo-Xu Cui received the B.S. degree from Department of Mathematics and Physics, Fujian University of Technology, Fuzhou, China in 2014. He is currently working toward his M.Sc. degree at School of Mathematics and Statistics, Wuhan University, Wuhan, China. His current research interests include image and signal processing, and statistical machine learning.

Qibin Fan received the B.S. degree in mathematics in 1982 and the M.Sc. degree in photogrammetry in 1988, from the Wuhan Technical University of Surveying and 


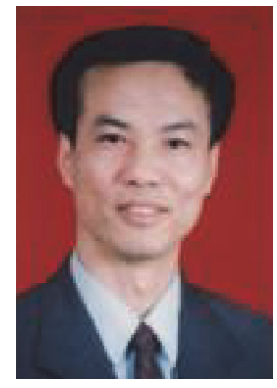

Mapping (WTUSM), and the Ph.D. degree in remote sensing and signal processing from Wuhan University in 2001.

He was an assistant professor from 1993 to 1997 and a Professor from 1997 to 2000 in the Department of Mathematics and Physics at WTUSM. Since August 2000, he has been a Professor in the School of Mathematics and Statistics at the Wuhan University. His research interests include wavelet analysis, sparse approximation, images restoration, and frame theory. 